\title{
Advanced Diagnostic Characterization of High-Power Hall Thruster Wear and Operation
}

\author{
George J. Williams, Jr. ${ }^{1}$ \\ The Ohio Aerospace Institute, NASA GRC, Cleveland, OH, 44135 \\ George C. Soulas, ${ }^{2}$ and Hani Kamhawi ${ }^{3}$ \\ NASA Glenn Research Center, Cleveland , OH, 44135
}

\begin{abstract}
Optical emission spectroscopy is employed to correlate $\mathrm{BN}$ insulator erosion with highpower Hall thruster operation. Specifically, actinometry leveraging excited xenon states is used to normalize the emission spectra of ground state boron as a function of thruster operating condition. Trends in the strength of the boron signal are correlated with thruster power, discharge voltage, and discharge current. In addition, the technique is demonstrated on metallic coupons embedded in the walls of the HiVHAc EM thruster. The OES technique captured the overall trend in the erosion of the coupons which boosts credibility in the method since there are no data to which to calibrate the erosion rates of high-power Hall thrusters. The boron signals are shown to trend linearly with discharge voltage for a fixed discharge current as expected. However, the boron signals of the higher-power NASA 300M and NASA 457Mv2 trend with discharge current and show an unexpectedly weak to inverse dependence on discharge voltage. Electron temperatures measured optically in the nearfield plume of the thruster agree well with Langmuir probe data. However, the optical technique used to determine $T_{e}$ showed unacceptable sensitivity to the emission intensities. Near-field, single-frequency imaging of the xenon neutrals is also presented as a function of operating condition for the NASA 457 Mv2.
\end{abstract}

\section{Nomenclature}

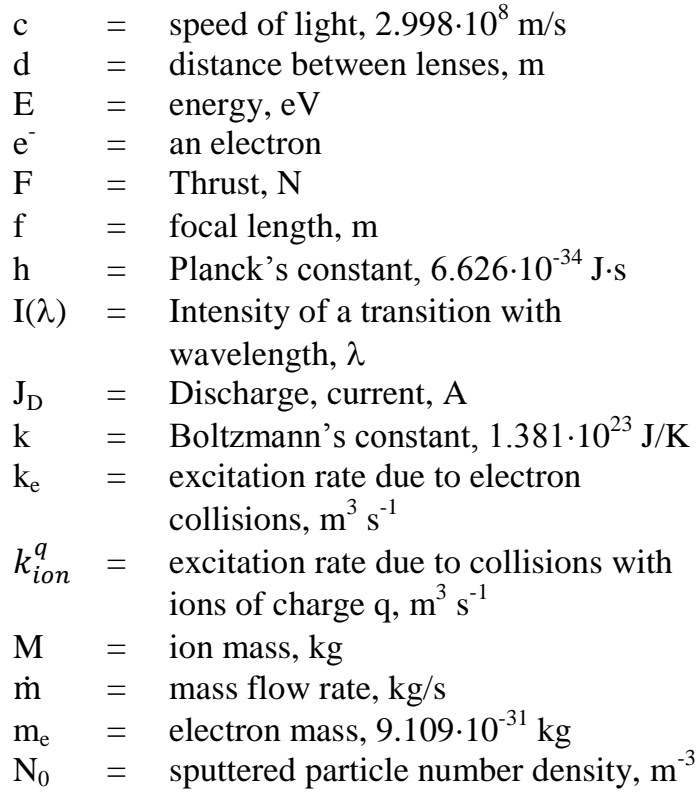

$$
\begin{array}{lll}
\mathrm{N}_{\mathrm{B}} & =\begin{array}{l}
\text { normalized signal strength for } \\
\text { the boron transition }
\end{array} \\
\mathrm{N}_{\mathrm{e}} & =\text { electron number density, } \mathrm{m}^{-3} \\
\mathrm{q} & =\text { ion charge } \\
\mathrm{S} & =\text { Amplification factor } \\
\mathrm{T}(\lambda) & =\text { Optical transmission function } \\
\mathrm{T}_{\mathrm{e}} & =\text { electron temperature, } \mathrm{eV} \\
\mathrm{V}_{\mathrm{D}} & =\text { discharge voltage, } \mathrm{V} \\
\mathrm{X} & =\text { axial distance from a focusing } \\
\mathrm{Xe} & =\text { lens, } \mathrm{m} \\
\alpha & =\text { denon ion of charge } \mathrm{i} \\
\alpha & \\
\lambda & =\text { wavelength, } \mathrm{m} \\
\eta_{\mathrm{A}} & =\text { Anode efficiency } \\
\mathrm{v} & =\text { frequency, } \mathrm{s}^{-1}
\end{array}
$$

\footnotetext{
${ }^{1}$ Senior Scientist, OAI, Propulsion and Propellants Branch, 21000 Brookpark Rd., MS 16-1, Associate Fellow.

2 Aerospace Engineer, Propulsion and Propellants Branch, 21000 Brookpark Rd., MS 16-1, AIAA Member.

${ }^{3}$ Aerospace Engineer, Propulsion and Propellants Branch, 21000 Brookpark Rd., MS 301-3, Associate Fellow.
} 


\section{Introduction}

$\mathrm{N}$ ASA's Hall-effect thrusters (HETs) are being designed to higher fidelity to support near term missions. Advanced modeling capabilities are enabling optimization of thruster design in terms of both performance and service life. In general, demonstration of thruster performance is straightforward and correlation of performance anomalies to design trades can be accomplished via standard physical probes located in the far to mid-plume plasma of the thruster. However, life-assessment and the impacts small modifications have on the near-field plasma require near field measurements which are very difficult with physical probes. To the extent that this near-field information can be collected via optical diagnostics, it is beneficial to do so.

Hall thrusters are a type of electric propulsion (EP) thruster with performance that is well suited for a number of future NASA space missions. Low power $(<1 \mathrm{~kW})$ Hall thrusters enhance and enable a number of small body and Radioisotope EP (REP) class science missions. Medium power ( 3.5-4.5 kW) Hall thrusters enhance and enable many NASA Discovery and New Frontier class science missions, while 4.5-10kW EP systems would be most applicable to NASA New Frontiers and Flagship class missions. High power ( $>20 \mathrm{~kW})$ EP systems are enabling and enhancing for time critical missions or missions requiring transportation of large payloads. A number of mission studies were performed highlighting the enhancing and enabling features of high power EP systems for reusable space tug applications for the transfer of payloads from LEO to GEO and for use in Mars mission scenarios. ${ }^{1,2,3}$

Several investigations have used optical emission spectroscopy (OES) to characterize the erosion of the BoronNitride (BN) insulators in HETs. ${ }^{4,5,6,7,8,9,10,11,12}$ Demonstrated validity, the extent to which the OES signal represents the erosion rate, remains the greatest challenge to OES as an erosion diagnostic. The emission signal is integrated over its collection volume and this leads to a lack of spatial resolution and to the possibility of detecting the same particles multiple times depending on sputter rate and plasma conditions. To some extent this can be mitigated through the use of spatial filters and multiple collection volumes. Even if the monitored transition is a ground-state transition of the sputtered material, the signal strength is proportional not only to the number density of the species, but also to the excitation rate of the sputtered atoms which is a function of the local plasma. As discussed below, electron excitation cross-sections of boron and other materials of interest to life assessment of electric propulsion systems are largely unknown. Moreover, even if these cross-sections are known, the electron temperatures in the regions of interest are extremely difficult to measure and are largely inferred from models. This precludes even the application of simple corona models to evaluate the relative signal strengths. In addition, sputtered atoms are excited to a number of states. Most of these are transitions to and from the ground state, and several different techniques have been proposed to correlate the number of atoms excited to one particular state to the total number of sputtered atoms. Finally, even if this can be resolved through an analysis of the relative excitation cross-sections, the diffuse nature of the sputtered material makes determination of the very local erosion rate via OES appear nearly impossible to determine. An alternative approach is incorporated in this investigation which leverages the local plasma via actinometry.

The development of the diagnostic capability at GRC is part of a larger effort to incorporate advanced diagnostics to facilitate design and demonstration of advanced HETs. The ultimate goal is to demonstrate and integrate a non-intrusive diagnostic capability that provides a real-time erosion measurement that mitigates or eliminates the need for very long duration testing of developmental hardware (at least from a life-limiting wear perspective). The technique will have the potential to measure not only insulator erosion but also cathode surface erosion and efflux of cathode emitter material. It may also have the ability to characterize the interaction of the thruster plume with spacecraft surfaces. The following discusses the first phase in the development of this capability which now has the capability to provide a real-time assessment of the relative insulator erosion rate with thruster operating condition.

\section{Experimental Hardware}

\section{A. Hall Effect Thrusters}

The evaluation and demonstration of the OES erosion monitoring technique took advantage of opportunities to test with different Hall Effect Thrusters. In addition to providing erosion data on these different thrusters, the ability to use the technique with a variety of thrusters of significantly different powers was demonstrated. One objective of this investigation was to compare the erosion trends of the small, albeit high-voltage HiVHAc thruster with higher power ones. All thrusters operated on xenon propellant.

The NASA-457Mv2 is the second version of a $50 \mathrm{~kW}$-class thruster whose development was initiated in 2000 for the NASA Space Solar Power Concept and Technology Maturation Program to enable space solar power 
systems and other high power spacecraft. ${ }^{13,14}$ The first laboratory version of the $50 \mathrm{~kW}$ thruster, designated NASA$457 \mathrm{M}$, had a $457 \mathrm{~mm}$ outer diameter ceramic discharge chamber and a centrally-mounted high current hollow cathode. It was fabricated and tested with xenon in 2002 at power levels up to $72 \mathrm{~kW}$ and at discharge voltages between 300 and $650 \mathrm{~V}$. Discharge specific impulses were measured from 1750 to $3050 \mathrm{~s}$ with anode efficiencies between $46 \%$ and $65 \%$ on xenon. ${ }^{13}$ The successful test campaign with the laboratory model NASA-457M led to the development of a higher fidelity version of this thruster, labeled the NASA-457Mv2. This newer version retained the same discharge chamber critical dimensions, however the magnetic circuit was designed for improved performance and reduced mass. In addition, the new mechanical design eliminated deficiencies with respect to anode mounting and electrical isolation, concentricity, and thermally induced mechanical interferences. Thruster fabrication was completed in 2004, but performance testing was not undertaken until 2011. ${ }^{17}$ Preliminary performance test results indicate that at a total thruster operating power of $25.2 \mathrm{~kW}$ and a discharge voltage of 500 $\mathrm{V}$, the NASA-457Mv2 generated $1.28 \mathrm{~N}$ of thrust and had a total thrust efficiency and specific impulse of $63 \%$ and $2,520 \mathrm{~s}$, respectively. For similar operating conditions $(26.3 \mathrm{~kW}$ and $500 \mathrm{~V})$, the NASA-457Mv2 generated $1.17 \mathrm{~N}$ of thrust had a total thrust efficiency and specific impulse of 55\% and 2,350 s. This preliminary performance characterization indicates that performance gains were attained with the NASA-457Mv2 when compared to the NASA-457M. ${ }^{15}$

The NASA-300M was designed and fabricated under the support of the ESMD Exploration System Research and Technology (ESR\&T) Program in 2005. The NASA-300M design is a scaled version of the NASA-457Mv2. ${ }^{15}$ The NASA-300M design incorporated lessons learned from the development and testing of the NASA-457M, NASA-400M, and NASA-457Mv2 thrusters. The goal of the design was to minimize thruster size while optimizing the magnetic field and plasma lens to attain improved performance. The NASA-300M nominal design specifications were a discharge power of $20 \mathrm{~kW}$, a discharge voltage range of up to $600 \mathrm{~V}$, a discharge current of up to $50 \mathrm{~A}$, and a magnetic circuit that has a magnetic field topology similar to the NASA-457Mv2. At $20 \mathrm{~kW}$ the thruster produced a peak thrust of $1.13 \mathrm{~N}$, a peak total thrust efficiency of $\sim 67 \%$ was achieved at a discharge voltage of $400 \mathrm{~V}$ and a peak total specific impulse of $2,916 \mathrm{~s}$ was demonstrated at a discharge voltage of $600 \mathrm{~V} .{ }^{15}$

The $300 \mathrm{M}$ and $457 \mathrm{Mv} 2$ were operated in Vacuum Facility 5 (VF5) at NASA GRC. VF 5 utilized a combination of diffusion pumps and cryo surfaces to maintain a pressure near the thruster less than $8 \cdot 10^{-5}$ Torr (corrected for Xe) during full power operation of either thruster. The operation leveraged a laboratory propellant feed system and power console, an inverted pendulum thruster stand, a data acquisition system, and several physical probes for plume interrogation. Detailed discussion of the testing of the $300 \mathrm{M}$ and $457 \mathrm{Mv} 2$ is available in the companion papers of Herman ${ }^{16}$ and Soulas. ${ }^{17}$

The NASA High-Voltage Hall Accelerator Engineering Model Thruster (HiVHAc EM, also referred to in more recent publications as the HiVHAc EDU1) is a 3.9-kW high-voltage Hall thruster. It is an advanced developmental thruster intended ultimately to provide an EP device with substantial cost and performance benefits when compared to the NASA Solar Electric Propulsion Technology Application Readiness (NSTAR) and NEXT ion engine systems for certain NASA Discovery class science missions. ${ }^{18,19}$ The EM thruster design incorporated the life extending channel replacement technology. In addition, the HiVHAc EM thruster was designed to survive structural and thermal environments for representative spacecraft and missions. The design leveraged all the experience, knowledge, and lessons learned during the development of the NASA-77M and AXFS NASA-103M.XL thrusters in addition to incorporating all of Aerojet's experience in manufacturing the flight qualified BPT-4000 Hall thruster propulsion system. A peak thruster efficiency of $\sim 59 \%$ was demonstrated when operating the thruster at a discharge voltage of $400 \mathrm{~V}$ at $3.5 \mathrm{~kW}$. A peak specific impulse of $2,720 \mathrm{~s}$ was demonstrated when operating the thruster at a discharge voltage of $700 \mathrm{~V}$ at $3.6 \mathrm{~kW} .^{15}$

For this investigation, the HiVHAc EM was operated in Vacuum Facility 8 (VF8) at NASA GRC. Magnetic field settings were selected to optimize performance. Cathode mass flow rate was fixed at $0.45 \mathrm{mg} / \mathrm{s}$, and a keeper current of $1.00 \mathrm{~A}$ was applied. Laboratory power supplies and feed system were used during this testing.

\section{B. Erosion Coupons}

The use of layers of imbedded materials to quantify the boron nitride (BN) erosion rate using OES was demonstrated by $\mathrm{Cho}^{8}{ }^{8}$ In that investigation, alternating layers of sputter deposited BN and silver (Ag) yielded a series of Ag OES peaks indicating when the layer of BN had eroded away. Layer thicknesses were not optimized and the data were somewhat inconclusive except to demonstrate the general feasibility of the technique. Coupons of

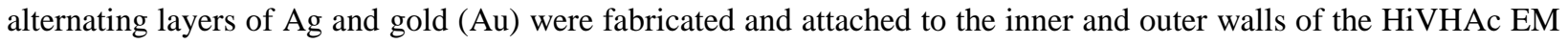
to address two concerns with the pure boron (B) OES in this investigation: is the relative change in OES signal level proportional to the erosion rate? and is the xenon (Xe) OES-based correction for $\mathrm{T}_{\mathrm{e}}$ reasonable? Table 1 compares the nominal volumetric sputter yields of $\mathrm{BN}, \mathrm{Au}$, and $\mathrm{Ag}$ as well as giving the energies of the excited states of 
interest and the associated wavelengths for OES. The energies associated with the excited levels from the ground state of $\mathrm{B}, \mathrm{Au}$, and $\mathrm{Ag}$ are similar. Therefore, as discussed below, the same normalization should be applicable to each species.

The erosion rate of the Au and Ag layers should vary with thruster operating condition due to changes in the energy and angle of incidence of the eroding ions. The OES signals $\mathrm{N}_{\mathrm{Au}}$ and $\mathrm{N}_{\mathrm{Ag}}$ (as defined below) should change proportionally, i.e. if the rate doubles, $\mathrm{N}_{\mathrm{Au}}$ and $\mathrm{N}_{\mathrm{Ag}}$ should double. The trends associated with the rates should be captured by the technique regardless of whether the metal layers erode similarly to the or at similar rates BN (which, given their matrices, is unlikely). For example, if diffusion or multiple excitation of the eroded species significantly impacts the collected OES signal, then the technique should show a disproportional relationship between the erosion rate of the metals and their OES signals.

$\mathrm{Au}$ and $\mathrm{Ag}$ foil were formed into square coupons of $3 \mathrm{~mm}$ sides and layers of $10 \mu \mathrm{m}$ thickness and stacked in alternating fashion seven layers thick. The $3 \mathrm{~mm}$ size corresponds roughly to the size of the focal spot of the optical probes on the insulator walls and is roughly three times wider than the target incorporated by Cho. However, the layers are roughly 100 times thicker than those of Cho. In part, the thicker layers are incorporated to extend the signal over several minutes to hours instead of tens of seconds. However, unlike Cho, the layers in this investigation were pressed to the BN surface and held by imbedded $\mathrm{W}$ wires. Thus, the sample materials extended into the discharge nearly $100 \mu \mathrm{m}$ at the start of the testing and were exposed on their upstream edges. Figure 2 shows a schematic of the coupon construction and a photograph of the coupons on the insulating surfaces prior to testing. One is located at the 9-o'clock position on the inner wall, and the other is at 3-o'clock on the outer wall. This allowed the optical probes to be placed on the same side of the thruster. Details of the probe arrangement are given below.

\section{Optical Probes and Cameras}

Optical probes were constructed using $2.5 \mathrm{~cm}$ diameter UV-silica lenses and flat windows, optical tubes, and SMA fiber optic connections. Lenses which matched the acceptance angle of the $400 \mu \mathrm{m}$ diameter UV-VIS fiber optic cables and protective windows were incorporated in all of the probes. For those probes focused on the BN surface, a second lens with a $30 \mathrm{~cm}$ or $50 \mathrm{~cm}$ focal length was incorporated in a threaded section which allowed adjustment of the focal point. Single-fiber, metal jacketed fiber optic cables coupled the probes to vacuum feed thrus and the feed thrus to a fiber optic multiplexer located external to the vacuum chamber. The probes were mounted near the thruster outside of a 45-degree exclusion zone.

Figure 3 shows schematically the arrangement of the probes with respect to each to the thrusters. For the $457 \mathrm{Mv} 2$, seven probes were used to interrogate the thruster and the near-field plume. Four probes were focused on the inner wall in $4.0 \mathrm{~mm}$ increments. These were intended to resolve the axial variation in $\mathrm{I}_{\mathrm{B}, 250}$. In order to place each probe roughly $30 \mathrm{~cm}$ from their point of interrogation, a complex arrangement of the probes was incorporated which is not reflected in the schematic. One probe was focused in the outer wall of the insulator near the exit plane (corresponding to probe 2 on the inner wall). Two probes which did not incorporate focusing lenses were used to interrogate the near field plasma 10 and $50 \mathrm{~mm}$ downstream. These probes incorporated spatial filters to insure that they collected light from a more or less cylindrical volume across the plume. Four probes were incorporated in $300 \mathrm{M}$ testing. One each was focused on the inner and outer insulator wall roughly $1.5 \mathrm{~mm}$ upstream of the exit plane. Two were used to interrogate the near field plume 8.0 and $18 \mathrm{~mm}$ downstream of the exit plane. Four probes were also used to interrogate the HiVHAc thruster which incorporated the metal coupons. One probe was focused on each of the coupons and the other two were focused at the same axial position but azimuthally off of the coupon. This arrangement allowed correlation of the B signal to that of the sample materials (assuming azimuthal symmetry).

The probes were calibrated before, during, and after each test. Standard Xe and W lamps were placed at the same distance as the location of measurement in the thruster for each probe and spectral data were recorded using the entirety of the probe-fiber-feed thru system before and after each series of testing. This measured the transmission function of the different probe assemblies which was used in the reduction of the data. Minor variations were noted which were likely the result of sputter deposition on the protective windows. The deposition tended to preferentially reduce the signal strength below a wavelength of $350 \mathrm{~nm}$. However, the degradation was negligible. Calibrations were performed during a test sequence by recording spectra at repeated thruster operating conditions. No significant changes in line intensities were noted, and the slight degradation of the UV signal was recorded and used in the normalization of the data.

Two digital cameras were placed in the exit plane of the 457Mv2 roughly $1 \mathrm{~m}$ away from the nearest edge of the discharge channel. The cameras imaged one half of the thruster and focused on the transverse center of the channel with an optical depth of about $1.5 \mathrm{~cm}$. This alignment enabled imaging of the plasma exiting the channel and 
cathode with a diffuse addition of signal from the rest of the plume. In order to image only neutral Xe, a filter centered at $473 \mathrm{~nm}$ with a FWHM of $1 \mathrm{~nm}$ was placed in front of one camera. A $541 \mathrm{~nm}$ filter with a FWHM of 10 $\mathrm{nm}$ was used to image the singly-charged ions. Although the $10 \mathrm{~nm}$ FWHM allowed some signal from other transitions, the Xe II signal at this wavelength is so strong that it dominates the collected light. Both filters were protected by fused-silica windows.

\section{Theory and Modeling}

In order to correlate the observed spectra to physical characteristics of the plasma or the erosion, it is necessary to relate the changes in the strengths of particular transitions to changes in the species or in plasma as a whole. Two models have been exercised. A simple collisional-radiative model allows correlation of the xenon spectra to plasma parameters and a hybrid corona model correlates the boron spectra to its concentration.

\section{A. Xenon Model}

A simple collisional radiative model has been exercised to correlate the xenon spectra to plasma properties. In general, the plasma processes relevant to Xe OES can be summarized in the following relations:

$$
\begin{gathered}
e^{-}+X e \rightarrow X e^{*}+e^{-} \\
e^{-}+X e^{+} \rightarrow X e^{+*}+e^{-} \\
e^{-}+X e^{*} \rightarrow X e^{+*}+e^{-} \\
e^{-}+X e \rightarrow X e^{* q+}+(1+q) e^{-} \\
X e^{+}+X e \rightarrow X e^{*}+X e^{+*} \\
X e^{2+}+X e \rightarrow X e^{*}+X e^{2+*} \\
X e^{2+}+X e \rightarrow 2 X e^{+*} \\
h v+X e \rightarrow X e^{*} \\
X e^{*} \rightarrow X e+h v \\
X e^{+*} \rightarrow X e^{+}+h v \\
X e^{*}+M \rightarrow X e+M+h v
\end{gathered}
$$

where a "starred" state is one that is simply of different internal energy (electron configuration) than the non-starred state. If relations 5-8 and 11 can be neglected, a corona model can be applied. It assumes all excitation occurs through collisions and all emission is governed by natural relaxation. Because there are several metastable states within the xenon spectra, a corona model cannot be applied reasonably without very high electron temperatures $\left(\mathrm{T}_{\mathrm{e}}\right.$ $>40){ }^{20}$

A model which includes all of these relations is considered to be a partial collisional-radiative model (CRM) since it includes both modes of population and depopulation of excited states. Unfortunately, CRMs require knowledge of the rate coefficients associated with these transitions over a large number of possible transitions. In general for xenon these are not known. Recent studies have experimentally determined the rate coefficients associated with Xe I and Xe II for a few transitions in the near infra red and in the visible spectrum. In particular, $\mathrm{Chiu}^{20}$ has developed effective excitation cross-sections $\left(\sigma_{\mathrm{e}}\right.$ and $\left.\sigma_{\text {ion }}\right)$ which experimentally convolve the possible transitions enabling a much simpler set of equations:

$$
\mathrm{k}_{\mathrm{e}}=\int_{0}^{\infty} 2 \mathrm{E} \sqrt{\frac{2}{\pi\left(\mathrm{kT}_{\mathrm{e}}\right)^{3} \mathrm{~m}_{\mathrm{e}}}} \exp \left(\frac{\mathrm{E}}{\mathrm{kT}_{\mathrm{e}}}\right) \sigma_{\mathrm{e}}(\mathrm{E}) \mathrm{dE}
$$

and 


$$
\mathrm{k}_{\mathrm{ion}}^{\mathrm{q}} \approx \sigma_{\mathrm{e}}(\mathrm{eV}) \sqrt{\frac{2 \mathrm{qeV}}{\mathrm{M}}}
$$

where $\mathrm{E}$ in Eqn 12 is the energy of the electron undergoing collision and $\mathrm{V}$ in Eqn 13 is the discharge voltage and $\mathrm{M}$ is the mass of a xenon atom. Equation 12 is numerically integrated given a table of or function for $\sigma_{\mathrm{e}}(\mathrm{E})$. Assuming quasineutrality, the intensity of a particular transition is then

$$
J(\lambda)=\frac{h c}{4 \pi \lambda} N_{0} N_{e}\left(k_{e}+k_{\text {ion }}^{1}+\frac{\alpha}{\sqrt{2}} k_{\text {ion }}^{2}\right)
$$

where $\alpha$ is the fraction of doubly charged ions. For the purpose of this investigation, $\alpha$ is assumed to be 0.20 . There is only modest sensitivity to this value for the $T_{e}$ of interest. The ratio of two lines is

$$
\frac{\mathrm{I}_{1}}{\mathrm{I}_{2}}=\frac{\left(\mathrm{k}_{\mathrm{e}}+\mathrm{k}_{\text {ion }}^{1}+\frac{\alpha}{\sqrt{2}} \mathrm{k}_{\text {ion }}^{2}\right)_{1}}{\left(\mathrm{k}_{\mathrm{e}}+\mathrm{k}_{\text {ion }}^{1}+\frac{\alpha}{\sqrt{2}} \mathrm{k}_{\text {ion }}^{2}\right)_{2}}
$$

Equation 15 can be used to either calculate $T_{e}$ or to estimate $I_{1}$ given $I_{2}$. The latter is of value if the rate coefficients are of different species in that, in principle, it could predict relative populations of the different states. Figure 3 shows $T_{e}$ as a function of ratio values of different Xe I line strengths. Note the asymptotic nature of the curves which restrict their validity to $\mathrm{T}_{\mathrm{e}}<20 \mathrm{eV}$.

\section{Boron Nitride Sputtering}

Neutral boron does not have a plethora of excited states like xenon. Since the ground state transition at $250 \mathrm{~nm}$ is populated almost exclusively by electron impact and depopulated by spontaneous emission, a corona model is justified. However, the excitation cross-section is unknown. Pagnon demonstrated that one can approximate the cross-section using actinometry to normalize the B OES signals. ${ }^{6}$ Actinometry uses the emission of one species (which is well-characterized) to correct the emission of another and is limited to applications with corona equilibrium and where the species have similar cross-sections, thresholds, and shapes as a function of electron energy. ${ }^{21}$

Following Pagnon, ${ }^{6}$ the upper state $5 \mathrm{p}^{1} \mathrm{D}^{0}(7 / 2)$ of the Xe II $(484 \mathrm{~nm})$ is populated principally by radiative decay from two ionic metastable states $5 \mathrm{~d}^{1} \mathrm{D}(7 / 2)$ and $5 \mathrm{~d}^{1} \mathrm{~F}(7 / 2)$. Therefore, the electrons that excite the $484 \mathrm{~nm}$ line (via the metastable states) have similar energy to those that excite the $250 \mathrm{~nm}$ boron line. The excitation of the two states should vary similarly with changes in the discharge. However, being an ion transition, the degree of ionization must also be accounted for. Pagnon does this by including the strength of the Xe I $(828 \mathrm{~nm})$ transition. In this investigation, the Xe I $(407 \mathrm{~nm})$ transition is used instead as its upper state is closer to ionization potential. All means of excitation including ion-atom collisions are included in this way - there is no need to separate them into specific rate coefficients.

The resulting expression for the amount of sputtered material, $\mathrm{N}_{\mathrm{B}}$, is

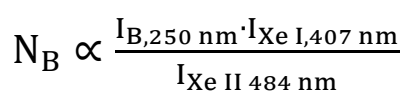

If $\mathrm{N}_{\mathrm{B}}$ at a particular operating condition can be tied to an insulator erosion rate, then $\mathrm{Eq} 16$ can be used to predict the erosion at other operating conditions.

Before the intensities of the lines were included in Eq 16 or any other comparative analysis, the raw signals were corrected for systematic perturbations or errors. These include offsets in the spectrometer baseline, transmission losses, and amplification through spectrometer sensitivity settings or data logging software. Equation 17 summarizes the correction of each raw signal:

$$
\mathrm{I}_{\lambda}^{\mathrm{cor}}=\left(\frac{\mathrm{I}^{\mathrm{raw}}-\mathrm{I}^{\text {offset }}}{\mathrm{s}}\right) \mathrm{T}(\lambda)
$$

Where $T(\lambda)$ is a self-normalized transmission function unique to each probe and $S$ is an amplification correction factor unique to each wavelength. As no "raw" data are presented, the superscript "raw" is dropped throughout this paper. "Uncorrected" data are those which have not undergone the normalization of Eq 16. 
Table 1 gives the ground state transitions of boron, gold, and silver monitored in this investigation. Note that the upper state energies of all of the transitions are comparable and, therefore, following the argument for selection of the Xe lines for correction of the B $250 \mathrm{~nm}$ line, can be normalized to plasma conditions using the same ratio of xenon lines. The volumetric sputter yields of $\mathrm{Au}$ and $\mathrm{Ag}$ due to Xe II impact are very similar in the $200 \mathrm{eV}$ to 300 $\mathrm{eV}$ energy range, roughly ten times that of BN. The differences in yields at $100 \mathrm{eV}$ potentially will give insight into the mean energies of the eroding ions. If the $\mathrm{Au}$ and $\mathrm{Ag}$ appear to sputter at significantly different rates, it can be inferred that most of the eroding ions have energies below $200 \mathrm{eV}$.

One of the limitations of OES when applied to thruster diagnostics is that it passively requires the excitation of target species within the zone of interrogation by electron collisions or other mechanisms. Figure 4 compares the electron excitation cross-section of the $250 \mathrm{~nm} \mathrm{~B} \mathrm{I}$ as a function of electron energy and the first-ionization crosssections of $\mathrm{B}, \mathrm{Xe}, \mathrm{Au}$, and $\mathrm{Ag} .{ }^{22,23,24,25}$ Note that the ionization cross-sections of $\mathrm{Xe}, \mathrm{Au}$, and $\mathrm{Ag}$ are nearly identical. This implies that the ionization fractions of these species should be similar (neglecting multiply charged ions). The cross-sections for B are smaller, but not greatly so, and are also of comparable magnitude to each other. Unfortunately, the wavelengths of the strongest of Au II and Ag II transitions are very close to Xe I and Xe II transitions making them very difficult to discern except in cases of extreme erosion. Data for the excitation crosssection of the $\mathrm{B}$ transition were not available above $30 \mathrm{eV}$, but were assumed to remain constant for electron energies less than $600 \mathrm{eV}$.

The degree to which the $\mathrm{B}$ atoms become ionized as opposed to excited depends on the electron energy distribution. Figure 5 compares self-normalized Maxwellian distributions for several electron temperatures. Also included in the Figure is a $20 \mathrm{eV}$ distribution which has a directed energy of $20 \mathrm{eV}$ as one might expect in the region of the Hall current. The mean free paths of excitation and ionization for $\mathrm{B}$ are comparable, several $\mathrm{cm}$, for all of these distributions, well within a factor of two, for electron number densities on the order of $10^{12} \mathrm{~cm}^{-3}$. Thus, the number density of B atoms is likely decreasing rapidly away from the walls and a larger fraction will leave the chamber as ions accelerated in the beam. Unfortunately, most of the strong B II lines lie close to weak Xe I or Xe II lines. For weak erosion, it is not possible to resolve the B II lines with the spectrometers used in this investigation. However, transients during thruster conditioning and magnetic field optimization have "revealed" the B II lines over the course of this investigation.

Correlation of the B I signal to the regression rate of the BN insulator is the ultimate aim of this investigation. However, due to restrictions associated with the transfer of sensitive information, actual and predicted erosion rates for these thrusters cannot be presented in an open forum. $\mathrm{N}_{\mathrm{B}}$ from Eq 16 is not, therefore, correlated in this presentation to projected or historical $\mathrm{BN}$ wear.

This restriction does not apply to the erosion rates of $\mathrm{Au}$ and $\mathrm{Ag}$ as measured in the configuration described above. Floating metallic surfaces will likely not have the same ion impingement as the electron emitting, dielectric BN. In addition, because the foils are raised slightly from the surface of the $\mathrm{BN}$, they may experience preferential erosion on their upstream surfaces. In addition, the crystalline structure of the metals will likely result in different differential sputter yields than BN. The resulting erosion rates of the coupons are not, then, representative of the erosion rate of the BN. Fortunately, that is not critical to this investigation. The foils responding differently to different plasma conditions associated with different thruster operating points is sufficient. The absolute erosion rate of the metallic surfaces may be determined either from the orderly transition from the OES signal of one species to another signifying the removal of an $\mathrm{Au}$ or $\mathrm{Ag}$ layer, or from the overall erosion as observed visually during the testing.

\section{E. OES Region of Interrogation}

As noted above, another of the limitations of OES is that light is collected from the entirety of the collection volume downstream of the probe. For cylindrically collimated probes such as those used in this investigation for measurement of plume properties, light is collected uniformly throughout the volume and, in principle, equal emission at any two points in the volume will impact the overall signal in an equal fashion. This is not the case for focused probes.

An estimate of the relative amount of the emission collected in the cone of interrogation of a focused probe can be made using ray matrices. ${ }^{26}$ The probes interrogating the interior of the HETs in this investigation consist of two thin lenses separated by a distance, $\mathrm{d}$, of $50 \mathrm{~mm}$. The off-centerline radius, $\mathrm{r}$, and the angle with respect to the line of interrogation, $\theta$, in the interrogation volume (condition 1) and those at the entry to the fiber optic (condition 2) are related by the following: 


$$
\left(\begin{array}{l}
r_{2} \\
\theta_{2}
\end{array}\right)=\left[\begin{array}{cc}
1 & f_{2} \\
0 & 1
\end{array}\right]\left[\begin{array}{cc}
1 & 0 \\
-\frac{1}{f_{2}} & 1
\end{array}\right]\left[\begin{array}{ll}
1 & d \\
0 & 1
\end{array}\right]\left[\begin{array}{cc}
1 & 0 \\
-\frac{1}{f_{1}} & 1
\end{array}\right]\left[\begin{array}{cc}
1 & X \\
0 & 1
\end{array}\right]\left(\begin{array}{l}
r_{1} \\
\theta_{1}
\end{array}\right)
$$

where $f_{1}$ is the focal length of the downstream lens, $f_{2}$ is the focal length of the lens coupling the light into the fiber, $\mathrm{d}$ is the distance between them, and $\mathrm{X}$ is the distance from the downstream lens to the point of interrogation. As a first approximation, in order for light to be collected by the fiber, the light reaching the fiber must be both within a given acceptance angle and within the radius of the fiber.

The relative fraction of collected light at any point between the focusing lens and just downstream of the focal point of the lens is shown in Fig. 6. The fraction is normalized to its maximum value for clarity of presentation. Note that this fraction is less than $10 \%$ throughout the majority of the cone. However, near the focal point, there is an extended region of relatively uniform collection. For the geometry of this investigation, the region of relatively uniform collection extends roughly $7.5 \mathrm{~cm}$. Figure 7 shows this weighted collection volume in the testing configuration of the NASA 300M. Note that the region of uniform collection extends through most of the plasma in the interrogation region. This is fortuitous since, although a small fraction of the light emitted at any point is collected throughout much of the collection volume, the total volume over which this occurs is much larger than the narrow part of the collection volume. $75 \%$ of the light collected would be from the larger region if the volume was emitting uniformly. As most of collection volume is outside of the plume, the probes actually collect light rather uniformly over a very narrow zone in the thrusters. This should allow for axial resolution of several $\mathrm{mm}$ near the insulator walls.

\section{F. Image Analysis}

Single-frequency imaging typically requires deconvolution of the image, often by Abel inversion. In this investigation, the cameras' depths of field were significantly smaller than the diameter of the thruster, roughly $5 \mathrm{~cm}$. Thus, a large fraction of the plume image was significantly out of focus and Abel inversion was not possible. Instead, images captured a segment of the plume which, for the $457 \mathrm{M}$, is essentially an average over a radial slice of the near-field plume.

At this preliminary stage, qualitative data can be obtained through image ratios. In principle, the electron temperature could be determined by taking images at the frequencies identified in the CR model above. However, filters for these wavelengths were not available with sufficiently narrow band pass FWHMs. These can be specially ordered, but were not pending results of this investigation. Instead, images of Xe I and Xe II lines are qualitatively compared to themselves, the B signal, near-field probe data, and other data. The latter two are referenced in the

discussion below as appropriate, however, note that the probe data were taken during the same test sequence as the OES data. ${ }^{16,}$

\section{G. Thruster Performance}

A standard measure of HET performance is the anode efficiency, $\eta_{\mathrm{A}}$. It is defined as

$$
\eta_{A}=\frac{F^{2}}{\dot{m} J_{D} V_{D}}
$$

and is a ratio of the power converted to thrust to the power input to the discharge, i.e. $J_{D} \cdot V_{D}$. All of the OES data were recorded for conditions where anode flows and magnetic fields were adjusted to optimize values of $\eta_{\mathrm{A}} \cdot{ }^{17}$

\section{Results}

\section{A. Actinometry Correction of B OES Signals}

Figures $8 \mathrm{a}$ and $8 \mathrm{~b}$ compare the raw, $\mathrm{I}_{\mathrm{B}, 250}$, and corrected, $\mathrm{N}_{\mathrm{B}}$, values for the $300 \mathrm{M}$ and $457 \mathrm{Mv} 2$ as a function of discharge power for discharge voltages of $250 \mathrm{~V}$ and $400 \mathrm{~V}$, respectively. Each curve is normalized to its maximum value - there is no comparison of rates between thrusters. Note that the correction is generally small. Also, there is, in general, a change from increasing the values to decreasing them as the discharge voltage increases for the same discharge power level. Because the curves are self-normalized, they are forced to agree at the highest powers of each curve. For reference, the ratios of different Xe II lines to the Xe I $497 \mathrm{~nm}$ line as a function of discharge power are given in Fig. 9. Note that the trends are different reflecting the different mechanisms with which the upper states are populated. 
In the discussion below the OES signal strengths are in terms of the intensities. For example. $\mathrm{I}_{\mathrm{Au}, 234}$ refers to the intensity of the $234 \mathrm{~nm}$ OES signal of Au I. The value of $\mathrm{I}_{\mathrm{B}, 250}$ which has been corrected by Eq 16 is referenced as the $\mathrm{N}_{\mathrm{B}}$ value. Other corrected signals also do not include the wavelengths, e.g. $\mathrm{N}_{\mathrm{Au}}$.

Uncertainty in the values of $\mathrm{N}_{\mathrm{B}}, \mathrm{N}_{\mathrm{Au}}$, and $\mathrm{N}_{\mathrm{Ag}}$ results from the signal to noise ratios their respective emission signals, noise and relative values of $\mathrm{I}_{\mathrm{Xe}} 407$ and $\mathrm{I}_{\mathrm{Xe+}, 484}$, the degree to which these lines fulfill the actinometry function, alignment, and contamination of the optics. For low levels of $\mathrm{I}_{\mathrm{B}, 250}, \mathrm{I}_{\mathrm{Au}, 243}$ and $\mathrm{I}_{\mathrm{Ag}, 328}$ the signal to noise ratios could be as low as 2 . However, by removing background and dark currents and digitally averaging the spectra before recording the intensity values, the ratios were increased to around 10 for most of the data, This still imparts a $\pm 10 \%$ uncertainty. In addition, $\mathrm{I}_{\mathrm{Xe} 407}$ is much weaker than $\mathrm{I}_{\mathrm{Xe}+, 484}$ and, especially at low thruster powers, the signal to noise ratio of $\mathrm{I}_{\mathrm{Xe}} 407$ was similar to that of $\mathrm{I}_{\mathrm{B}, 250}$. The uncertainty in $\mathrm{I}_{\mathrm{Xe}} 407$ was reduced by measuring it with both spectrometers and comparing trends. When the value of $\mathrm{I}_{\mathrm{Xe}} 407$ became very small with respect to $\mathrm{I}_{\mathrm{Xe+}, 484}$, in the spectra of the visible spectrum (which had to accommodate both), it was scaled with the value from the UV spectrum spectrometer which had the ability to resolve a much higher range of intensities. The $\mathrm{I}_{\mathrm{Xe}} 407$ signal was scaled to clean Xe I lines nearby on both spectrometers. This may have introduced another $10 \%$ uncertainty. As seen in Fig. 9, this was more of a factor at low powers as $\mathrm{I}_{\mathrm{Xe+}} 484$ tended to decrease with thruster power. The variation in signal due to the coating of optics was relatively small. The comparison of pre- and post-test transmission as well as the observation of repeated operating conditions tended to capture this slow trend in the reduction of the data. Unfortunately, there are insufficient data to assess an uncertainty on the validity of the actinometry assumptions themselves. Therefore, the overall uncertainty of the corrected values then varied from $\pm 10 \%$ for large signals to perhaps a factor of 2 at low signals. Normalization of the corrected values by the maximum values in a trend tended to preserve this relative error. Low signal strengths sometimes correlated with low powers, but also with location of measurement. As seen in the NASA 457Mv2 data below, resolution of the point of interrogation near the exit plane of the thrusters was not critical.

\section{B. OES Measurement of Sputter-Eroded Gold and Silver Coupons}

Sputter-eroded $\mathrm{Au}$ and $\mathrm{Ag}$ were observed from coupons on both the inner and outer channel walls of the HiVHAc EM thruster. $\mathrm{I}_{\mathrm{Au}, 243}$ and $\mathrm{I}_{\mathrm{Ag}, 328}$ trended with $\mathrm{I}_{\mathrm{B}, 250}$ and $\mathrm{I}_{\mathrm{B}+, 345}$ and not with the intensities of various Xe I or Xe II transitions during brief periods of off-nominal operation during startup, optimization of the magnetic field, and intentional ion impingement on either the inner or outer walls. Au I, Au II, Ag I, Ag II, B I and B II were observed during these periods of accelerated wear which confirmed wavelength selection for automatic recording of particular wavelengths over extended operation. Signal to noise ratios were high and data were recorded after digitally averaging 12 independent spectral scans of $400 \mathrm{~ms}$ each. This reduced the error in $\mathrm{I}_{\mathrm{B}, 250}$ in these very-low power measurements to less than that observed at $10 \mathrm{~kW}$ in the NASA 457Mv2. Nevertheless, it was still on the order of $30 \%$. Uncertainties in $\mathrm{I}_{\mathrm{Au}, 243}$ and $\mathrm{I}_{\mathrm{Ag}, 328}$ were roughly $50 \%$ and, because the Xe I transitions were relatively strong, correspond to the uncertainties in $\mathrm{N}_{\mathrm{Au}}$ and $\mathrm{N}_{\mathrm{Ag}}$. During nominal thruster operating conditions, Xe II and Xe III lines masked Au II and Ag II lines except the Au II line at $300 \mathrm{~nm}$. Figure 10 compares the trends observed in $\mathrm{N}_{\mathrm{Au}}$ and $\mathrm{N}_{\mathrm{Ag}}$, with discharge power. The open symbols with dashed lines correspond to the signal from the outer wall. Even though the outer wall values are higher, the data are normalized to the peak of the inner wall value to be consistent with the convention followed throughout the rest of the paper. For comparison, also shown are $\mathrm{N}_{\mathrm{B}}$ values collected by the probes imaging the regions next to the coupons, cf Fig. 3c. Note that here, as in most of the data below, the signal strengths of the material sputtered from the inner wall are higher. Also, the ratio of initial to final values of $\mathrm{N}_{\mathrm{Au}}$ and $\mathrm{N}_{\mathrm{Ag}}$ are higher than those for $\mathrm{N}_{\mathrm{B}}$. This suggests that, as expected, the erosion processes associated with the coupon erosion are not the same as those of the BN wall itself.

The absolute erosion rate of the individual foils of the metallic coupons is difficult to quantify. An initial period of about $300 \mathrm{~s}$ in which there was a clear $\mathrm{I}_{\mathrm{Au}, 243}$ signal from the inner wall with no $\mathrm{I}_{\mathrm{Ag}, 328}$ signal suggests that some fraction of the $10 \mu \mathrm{m}$ thick outer foil eroded over this time. However, when the Ag I signal became clear, the Au I signal did not disappear, indicating that at least some of the $9 \mathrm{~mm}^{2}$ surface remained. Indeed, there was very little change observed in $\mathrm{I}_{\mathrm{Au}, 243}$ suggesting that most of the Au remained. Data were collected alternatively between the inner and outer walls. While this transition from Au only to Au and Ag signals on the inner wall was monitored, no data were collected from the outer wall which likely was undergoing a similar transition. There were relative fluctuations in $\mathrm{I}_{\mathrm{Au}, 243}$ and $\mathrm{I}_{\mathrm{Ag}, 328}$ for a fixed thruster operating point, but these did not lend themselves to quantitative characterization of the erosion rates.

During the testing, images were taken of the HiVHAc EM's discharge which revealed some information regarding the quality of the coupons on both the inner and outer walls (cf Fig. 2b). The exposed fractions of Au or $\mathrm{Ag}$, were not possible to determine, but the overall size of the coupon was quantifiable. The coupons on both the inner and outer walls eroded from the upstream side in a relatively uniform pattern. This suggests that either edge 
effects, or directed ions, or both accelerated the wear of the upstream edges of the foil. Post-test analysis of the images resolved the relative sizes of the coupons to within 5\%. Figure 11 compares the integrated $\mathrm{N}_{\mathrm{Au}}$ and $\mathrm{N}_{\mathrm{Ag}}$ values and the eroded fraction of the coupon on the outer wall of the HiVHAc EM thruster as a function of time over the duration of the test. Note that there is qualitatively good agreement. No error bars are placed on the fraction of coupon remaining because there is no estimate of coupon thickness which was likely thinning during testing. Indeed, there was a period of about $750 \mathrm{~s}$ between $3700 \mathrm{~s}$ and $4500 \mathrm{~s}$ that showed significant increases in the OES signals. This period was also likely responsible for the $15 \%$ decrease in size of the coupon (no image was taken immediately before the period). The merging of the trends after $8500 \mathrm{~s}$ results from a combination of the increased erosion at higher $\mathrm{V}_{\mathrm{D}}$ and of the normalization of the curves to 1 at the end of test.

Figure 12 shows the trends associated with the inner wall. Again, there is reasonably good agreement. Note that the values of integrated $\mathrm{N}_{\mathrm{Ag}}$ and of the coupon's eroded fraction are slightly lower in Fig. 12. The differences are within the uncertainty of the data. Trends for the integration of the combined $\mathrm{N}_{\mathrm{Au}}$ and $\mathrm{N}_{\mathrm{Ag}}$ signals and of other $\mathrm{N}_{\mathrm{Au}}$ and $\mathrm{N}_{\mathrm{Ag}}$ signals for different wavelengths yielded nearly identical curves. While the data did not yield the clear erosion rates and signal cutoffs that were desired, the ability of the OES technique to capture the overall trend in erosion rate of the samples is encouraging. It suggests that the actinometry technique might enable the OES of sputtered B to be a reasonable in-situ erosion diagnostic.

\section{OES Measurement of Sputter-Eroded Boron}

Optical emission spectra of sputtered B were collected on the HiVHAc EM, NASA 300M, and NASA 457Mv2 thrusters in three separate sets of tests as noted above. In all cases, there was a characterization of the B sputtered from the near-exit plane region of the inner and outer BN insulator walls. Data were collected over a wide range of discharge powers and for several $\mathrm{J}_{\mathrm{D}}$ and $\mathrm{V}_{\mathrm{D}}$ combinations at several constant discharge powers. Data were also collected at off-nominal magnet current settings and facility pressures. These data are not presented here. However, real-time correlations between the magnet current settings and the B OES signal were clear.

Figures13a-c compare $\mathrm{N}_{\mathrm{B}}$ values for the inner and outer walls as a function of discharge power for the HiVHAc EM, NASA 300M, and NASA 457Mv2, respectively. The signals are taken roughly $1.5 \mathrm{~mm}$ of the exit plane in each case. Figure 13a shows a linear variation of $\mathrm{N}_{\mathrm{B}}$ with discharge power for a fixed discharge current of $5 \mathrm{~A}$. The difference in $\mathrm{N}_{B}$ values of the inner and outer walls lay within the uncertainty of the values themselves. In this investigation, the HiVHAc EM was only operated at a fixed current. The $\mathrm{N}_{B}$ values associated with the inner and outer walls of the NASA 300M over a wide range of discharge voltages and currents show distinct separation which increases with discharge power in Fig. 13b. Figure 13b also indicates a second-order dependence of $\mathrm{N}_{B}$ on discharge power. The data associated with one $V_{D}$ or one $J_{D}$ do not lay outside of the general trend. This was not the case for the NASA 457Mv2 as is seen in Fig. 13c. Note that there are two distinct trends of $\mathrm{N}_{\mathrm{B}}$ with discharge power for each of the two $V_{D}$ shown. Each trend is more linear than the trend shown $n$ Fig. 13b. $N_{B}$ values for operation at $\mathrm{V}_{\mathrm{D}}=300 \mathrm{~V}$ lay near the curve for $\mathrm{V}_{\mathrm{D}}=250 \mathrm{~V}$. Figure $13 \mathrm{c}$ suggests that at the same power level, operation at higher $\mathrm{V}_{\mathrm{D}}$ results in lower erosion which is counter-intuitive.

Figure 14 shows $\mathrm{N}_{B}$ values as a function of axial position along the inner wall of the NASA $457 \mathrm{Mv} 2$ as a function of $\mathrm{J}_{\mathrm{D}}$ for $20 \mathrm{~kW}$ operation. Each of the axial locations corresponds to data from one of the four OES probes aimed at the inner wall (cf Fig. 3a). Data shown in Figures 14-18 are normalized to the peak $N_{B}$ value for all cases. The relative erosion rates associated with the positions and operating conditions can then be inferred. Note that there is a monotonic increase in $\mathrm{N}_{\mathrm{B}}$ values with current and that the values increase towards the exit of the thruster $(\mathrm{x}=0)$. The probes narrow collection volume appears to have allowed reasonable axial resolution of $\mathrm{I}_{\mathrm{B}}$. For all of the data, there was little variation in $\mathrm{N}_{\mathrm{B}}$ within $5 \mathrm{~mm}$ of the exit plane. Two measurements suggest the the flow of $\mathrm{B}$ from upstream is negligible on the downstream OES signals downstream. One is that even though the collection volumes of all four probes pass through roughly the same region of the discharge and only become distinct near the wall, they record significantly different $I_{B}$ values. This is despite the fact that, to some degree, the collection volumes cross in order achieve the necessary alignment without positioning the probes prohibitively far downstream of the exit plane. The second was measurement of the $\mathrm{I}_{\mathrm{B}, 250}$ and $\mathrm{I}_{\mathrm{B}+, 300} 2 \mathrm{~mm}$ downstream of the exit plane in a few cases. The intensities remained below the detection threshold for all operating conditions. This suggests that the B was being lost to ionization and acceleration but also that there was not a large cloud of slow-moving B which is impacting the measurements.

Figure 15 shows $N_{B}$ values as a function of axial position for $25 \mathrm{~kW}$ operation. Note that the curves for $J_{D}=83$ $A$ and $J_{D}=100$ A lay very close together. The $N_{B}$ values for $25 \mathrm{~kW}$ did not show the more or less linear dependence on $J_{D}$ as for $20 \mathrm{~kW}$. This trend is more pronounced in the $30 \mathrm{~kW}$ data shown in Fig. 16 in which the $\mathrm{N}_{\mathrm{B}}$ values for $\mathrm{J}_{\mathrm{D}}=100$ A lay below those for $\mathrm{J}_{\mathrm{D}}=86 \mathrm{~A}$. Those for $\mathrm{J}_{\mathrm{D}}=60 \mathrm{~A}$ and $75 \mathrm{~A}$ lay very close together. The latter is similar to the trend see in Fig. 14, but the former suggests that $V_{D}$ has a larger impact on erosion than $J_{D}$ for higher 
powers. While this trend with $V_{D}$ is more intuitive than the trend seen in Fig. 13b with the NASA 300M, the overall efficiency of the thruster is less at these higher currents at higher powers. ${ }^{17}$ It is also not the trend seen in Fig. 17 for $40 \mathrm{~kW}$ operation. In Fig. 17, $\mathrm{N}_{\mathrm{B}}$ values are proportional to $\mathrm{J}_{\mathrm{D}}$ at $80 \mathrm{~A}$ and $100 \mathrm{~A}$. These values are also significantly less than they were for $30 \mathrm{~kW}$ and comparable to those for $25 \mathrm{~kW}$.

Figure 18 compares $\mathrm{N}_{\mathrm{B}}$ values for $\mathrm{J}_{\mathrm{D}}=100$ A for several discharge powers. These curves suggest that the erosion will decrease with power for 100 A operation and that operation at higher $\mathrm{V}_{\mathrm{D}}$ does not necessarily imply decreased service life. The $\mathrm{N}_{B}$ values associated with $\mathrm{J}_{\mathrm{D}} \approx 80 \mathrm{~A}$ are roughly independent of power for 20,25 , and 30 $\mathrm{kW}$ and only decrease for the $40 \mathrm{~kW}$ data shown in Fig. 17. This trend also suggests that $\mathrm{J}_{\mathrm{D}}$ is more significant than $\mathrm{V}_{\mathrm{D}}$ in determining erosion.

For $V_{D}$ to play a greater role in determining the erosion rate than $J_{D}$, the $N_{B}$ values for a fixed $J_{D}$ should increase significantly with power (i.e. with $\mathrm{V}_{\mathrm{D}}$ ). This trend is not seen in any of the NASA 457Mv2 data except for the case of $86 \mathrm{~A}$ at $30 \mathrm{~kW}$. Indeed, the opposite trend was generally observed. Figures 18 and 19 shows the trend in $\mathrm{N}_{\mathrm{B}}$ values with $J_{D}$ and $V_{D}$, respectively for a range of operating conditions of the $457 \mathrm{Mv} 2$. The $\mathrm{N}_{B}$ values are normalized to the same value they are in the previous figures. Also plotted in Figs. 18 and 19 are the products of $J_{D}$ and $\left(1-\eta_{\mathrm{A}}\right)$ which are taken from Soulas. ${ }^{17}$ The products of these values are normalized in the same fashion, i.e. by the largest value in the complete set of plotted in both figures. Note the trends in Fig. 18 are very similar suggesting that $N_{B}$ trends strongly with $J_{D}$ and that the slope is roughly $\left(1-\eta_{A}\right)$. Figure 19 shows that $N_{B}$ values decrease with increasing $V_{D}$ and follow the same trends with respect to $V_{D}$ as $J_{D}\left(1-\eta_{A}\right)$. Figures 20 a and $20 b$ show $N_{B}$ values as a function of $V_{D}$ for $J_{D}=25$ A and 40 A, respectively, for the NASA 300M. These figures show that $N_{B}$ values for this thruster had a weak dependence on $V_{D}$. As in Fig. 13b, the $N_{B}$ values from the inner wall are significantly higher than those from the outer wall.

\section{Plasma Measurements}

Calculation of $\mathrm{T}_{\mathrm{e}}$ using ratios in Eq 15 was attempted for probes imaging the $\mathrm{BN}$ walls (i.e. interior to the thrusters) and those imaging downstream of the thrusters' exit planes. The probes used to determine $\mathrm{N}_{\mathrm{B}}$ values yielded Xe I line ratios outside of the ranges which could be used to accurately estimate $T_{e}$. This is consistent with several models and internal probe measurements which suggest $T_{e}>40 \mathrm{eV}$ for most operating conditions. It points to the strength of using Eq 16 for determining $\mathrm{N}_{\mathrm{B}}$ which does not depend on explicitly knowing $\mathrm{T}_{\mathrm{e}}$, but rather on simply capturing its effect on the populations of complementary states. However, Eq. 15 did yield reasonable numbers for data taken downstream of the thruster exit planes.

Uncertainty in the calculation of $T_{e}$ resulted from the signal to noise of the intensities of the Xe I transitions as well as the error associated with the extraction of $T_{e}$ from Eq 15. The ratio of intensities $I_{X e, 462} / I_{X e, 473}$ was found to offer the best relative signal to noise values over the range of operating conditions tested with the thrusters. Nevertheless, the ratio itself varied by as much as $10 \%$ at many of the operating conditions. This was sufficient to preclude the use of $\mathrm{Eq} 15$ for $\mathrm{T}_{\mathrm{e}}$ greater than $30 \mathrm{eV}$. The variation introduced an uncertainty which itself varied with the value of $\mathrm{T}_{e}$ For $\mathrm{T}_{\mathrm{e}}<5 \mathrm{eV}$, the error was around 5\%. For $5<\mathrm{T}_{\mathrm{e}}<10 \mathrm{eV}$, the error was $10-20 \%$, for $10<\mathrm{T}_{\mathrm{e}}<20$ $\mathrm{eV}$, the uncertainty increased to $50 \%$ and for $\mathrm{T}_{\mathrm{e}}>20 \mathrm{eV}$, the uncertainty was greater than a factor of 2 . For most of the data discussed below, a 50\% uncertainty is assumed for the calculated $\mathrm{T}_{\mathrm{e}}$.

Figure 21 compares the calculated electron temperatures $18 \mathrm{~mm}$ downstream of the NASA 300M exit plane assuming different Xe-Xe collision terms. Note that the inclusion of the Xe-Xe collisions with an $\alpha$ of 0.25 results in a significantly higher $T_{e}$ for these points. Langmuir probe data were collected over a wide range of locations downstream of the thruster, ${ }^{16}$ including close to where the OES plume data were collected. The probe measured a $T_{e}$ of $17 \mathrm{eV}$ at $20 \mathrm{~kW}\left(\mathrm{~V}_{\mathrm{D}}=300 \mathrm{~V}\right)$ which is in remarkable agreement with the OES calculation including the collision terms. All calculations below include these terms, regardless of line ratios used. An $\alpha$ of 0.25 is assumed for all operating conditions.

Figure 23 compares the electron temperature at two axial locations as a function of discharge power and double to single ion current using the ratio of $462 \mathrm{~nm}$ and $473 \mathrm{~nm}$ Xe I lines. Why the peak in $\mathrm{T}_{\mathrm{e}}$ at $10 \mathrm{~kW}$ at $8 \mathrm{~mm}$ downstream is not present $18 \mathrm{~mm}$ downstream remains unclear. However, the same trend is observed when other line ratios are used. The higher $\mathrm{T}_{\mathrm{e}}$ at 7 to $15 \mathrm{~kW}$ may result from the change in location of the "core" of the plasma exiting the thruster. These data will be correlated with high-resolution near-field imaging below.

Figure 24 compares $T_{e}$ values calculated using the ratio of $\mathrm{I}_{\mathrm{Xe}, 462} / \mathrm{I}_{\mathrm{Xe}, 473}$ in the plume of the NASA 457Mv2 thruster for $\mathrm{V}_{\mathrm{D}}=500 \mathrm{~V}$ operating conditions. Note that a point was taken $2 \mathrm{~mm}$ downstream for the low power case, and, as expected it shows a significantly higher $T_{e}$ than values farther downstream. While this is consistent with the OES values for lower powers, it suggests that there is another reason that the ratios are not yielding useful data. At $50 \mathrm{~mm}$, one would expect $\mathrm{T}_{\mathrm{e}}$ to be significantly less than $30 \mathrm{eV}$ as was measured by the plasma probes. 
Why there were such large fluctuations in the OES data which precluded calculation of $T_{e}$ in the plume for most operating conditions is unclear.

Figure 25 compares $T_{e}$ values calculated using the same ratio of Xe I lines in the plume of the NASA 457Mv2 thruster for $\mathrm{V}_{\mathrm{D}}=400 \mathrm{~V}$ operating conditions. The values at $10 \mathrm{~mm}$ show a rise in the region around $20 \mathrm{~kW}$ similar to that seen in the NASA $300 \mathrm{M}$ data at $13 \mathrm{~kW}$. As with the NASA $300 \mathrm{M}$ data, the rise is lost farther downstream. Probe measurements at $50 \mathrm{~mm}$ at the $30 \mathrm{~kW}$ point show a $\mathrm{T}_{\mathrm{e}}$ of $12 \mathrm{eV} .{ }^{16}$ Variations in the data at $6 \mathrm{~kW}$ reflect changes in the facility background pressure which was varied intentionally to determine its effects. ${ }^{17} \mathrm{~T}_{\mathrm{e}}$ were higher for higher facility pressures which may suggest that the acceleration region of the plume was advancing downstream, but it is highly unlikely that it extended to $50 \mathrm{~mm}$ downstream. Perhaps the increase in charge exchange ion production significantly altered the distribution of electronic states among the Xe I atoms and this yielded an artificially high $\mathrm{T}_{\mathrm{e}}$.

\section{E. Single Frequency Images}

Figure 26 shows two images of the near-field plume of the NASA 457Mv2. Figure 24a is taken with a $473 \mathrm{~nm}$ pass filter which, as noted above, corresponds to a Xe I transition used in the calculation of $\mathrm{T}_{\mathrm{e}}$. Conveniently, it is relatively isolated from Xe II and Xe III emission lines. Figure 24b is taken with a $541 \mathrm{~nm}$ pass filter which corresponds to a strong Xe II emission line. Figure 24b, and all images taken with this filter suffered from etaloning which made analysis difficult.

Figure 27 compares Xe I images taken of the NASA 400Mv2 operating at $V_{D}=400 \mathrm{~V}$ at four different $J_{D}$. The images show the region roughly 3 channel widths downstream (the numbers on the axes refer to pixels). This extends through the region of both OES probes shown in Fig. 23. For a constant $\mathrm{T}_{\mathrm{e}}$ around $50 \mathrm{~mm}$ downstream, changes in the emitted signal should be proportional to the density of atoms with this excited state. This would allow calculation of the number density of Xe I given the C-R model outlined above. However, $T_{e}$ was shown to not be constant in the very near field which corresponds to the regions of highest signal. Because of the ambiguity of this transition, further reduction of these images in terms of $\mathrm{T}_{\mathrm{e}}$ or $\mathrm{n}_{\mathrm{Xe}}$ is not reported here. The wing in the image extending towards the cathode (right in the image) appears in part as a result of the inclusion of signal from the out of focus region of the remainder of the channel. However, it could also signal a region of high $\mathrm{T}_{\mathrm{e}}$. Neutral density measurements made via optical imaging with Abel inversion on a thruster simulator in the same region show a similar distribution. ${ }^{27}$ Jameson's data showed a strong peak in signal near the channel exit confined more or less to the channel cross-section and then a trend towards higher density towards centerline farther from the exit plane. At a point several millimeters downstream, the density was shown to be the same in this wing as it is in front of the channel. ${ }^{27}$ This is similar to trends shown in Figs 25 and 26. Probe data suggest that $T_{e}$ varies weakly from 0.2 to 0.5 thruster diameters downstream across the plume. ${ }^{16}$ In this regard, those data at $400 \mathrm{~V}, 75 \mathrm{~A}$ resemble the shape of Fig. 25b more closely than of Fig. 25c. Additional probe data or imaging at another frequency are required to demonstrate this possibility.

Figure 28 compares the Xe I images for the NASA 457Mv2 operating at $100 \mathrm{~A}$ at four different discharge voltages. There is likely much more charge exchange in the $200 \mathrm{~V}$ plume than in the $500 \mathrm{~V}$ plume both because of the higher number of beam ions and the higher number of background neutrals. Therefore, that the Xe I plume would be more pronounced at the higher voltage is unexpected. As with Fig. 25, Fig. 26 requires additional information in order to provide a measure of densities or $\mathrm{T}_{\mathrm{e}}$. However, the images should lend themselves to that analysis.

\section{Discussion}

\section{A. Insulator Erosion}

The trends of HET insulator erosion at higher powers appears to be more dependent on anode flow rate than $\mathrm{J}_{\mathrm{D}}$ or $V_{D}$. The largest $N_{B}$ values are seen for high currents at low voltages. Assuming the $\mathrm{N}_{B}$ values are proportional to the erosion rates, this trend is not consistent with the dependence observed at lower powers observed by many investigations. The $\mathrm{BN}$ erosion rates observed in most studies show that the rate is proportional to the discharge power. Thus, higher currents at lower voltages would erode less than higher currents at higher voltages. In fact, there continues to be a debate in the community about which is dominant, $J_{D}$ or $V_{D}$. The credibility of the measurements reported in this investigation would be highly in question had not the same technique reproduced the expected trends in low power HiVHAc EM thruster operation. For the HiVHAc EM thruster, $\mathrm{N}_{B}$ was only a function of $V_{D}$ as $J_{D}$ was held constant. This trend is consistent with previous investigations on low-power thrusters which show a linear dependence of the erosion rate on $\mathrm{V}_{\mathrm{D}} \cdot{ }^{9,10}$ 
In addition, the technique reasonably captured the trend in erosion of coupons placed on the wall of the HiVHAc EM thruster as the thruster's power was varied. The OES technique captured transients in real-time on multiple thrusters which were consistent with expectations of high-erosion events, and, at times, provided an indicator of the onset of such transients which led to their mitigation. Finally, while not discussed in detail beyond Fig. 8, it should be noted that $\mathrm{I}_{\mathrm{B}, 250}$ trended with $\mathrm{J}_{\mathrm{D}}$ in much the same manner as $\mathrm{N}_{\mathrm{B}}$ throughout the data. And, note, OES of $\mathrm{I}_{\mathrm{B}, 250}$ as a predictor of low-power HET erosion has been validated previously through weight loss measurements. ${ }^{9}$

There are several other potential explanations as to why the $\mathrm{N}_{\mathrm{B}}$ values trend as they do at higher powers. One is that the erosion is primarily due to lower-energy ions accelerated through sheaths and not due to primary ions. Differences in $\mathrm{Au}$ and $\mathrm{Ag}$ erosion rates might reveal an energy dependence of the erosion and thereby indicate the energy of eroding ions. However, the test design was not satisfactory to yield this data. This remains a possibility, then, and the higher- $\mathrm{T}_{\mathrm{e}}$ likely associated with high-power operation could generate a large number of ions upstream of the acceleration zone. Previous investigations have shown that the ionization and acceleration regions change with $\mathrm{V}_{\mathrm{D}}$. The acceleration zone lengthens and moves upstream. ${ }^{28}$ Perhaps the ionization region lengthens significantly at high flow rates. Both of these possibilities could be resolved with detailed internal probe measurements. Another possibility is that the B atoms are being either ionized at higher rates at higher voltages (higher $\mathrm{T}_{\mathrm{e}}$ ) or collisionally excited in high-density flows. The latter should be included in the actinometry, but the B atoms may be collisionally populated through additional paths. A higher ionization rate should be present, if not greater for lower power thrusters. None of these explanations is in itself satisfactory.

If the dependence on $J_{D}$ and independence on $V_{D}$ are in fact an erroneous trends, the most probable reason is that the Xe transitions being used to normalize $\mathrm{I}_{\mathrm{B}, 250}$ are being averaged over the entire collection volume of the probes and not just in the region of high B density. The B atoms may be in highest density near the walls, as is suggested by the ability to discern different $\mathrm{I}_{\mathrm{B}, 250}$ at different axial locations despite significant overlap of the OES collection volumes with the discharge plasma. However, the Xe density is strong throughout the chamber and varies significantly in $n_{e}$ and $T_{e}$ over the collection volumes. The similar trends in $I_{B, 250}$ and $N_{B}$ may reflect that the actinometry is not properly correcting the former. The changes in the discharge plasma of the small HiVHAc EM thruster with $V_{D}$ may not be similar to those of the larger thrusters and, therefore, the integrated Xe signals across the HiVHAc thruster capture the dependence on $V_{D}$ whereas those for the larger thrusters do not.

Demonstrating validity of the predictive capability remains the principle issue associated with OES measurements and additional testing is needed to eliminate some concerns. In the near future, absolute erosion measurements of $\mathrm{BN}$ will be correlated with $\mathrm{N}_{\mathrm{B}}$ values. This will hopefully be accomplished through a combination of long duration testing and coupon testing. In particular, coupons of BN separated by Au layers appears to be an attractive option. Recent internal probe measurements of the NASA $300 \mathrm{M}$ may help to validate this OES technique as well as numerical models. Modeling and measurement of the ionization processes will also build credibility in the technique.

\section{B. Plasma Characterization}

Electron temperatures calculated from the ratio of Xe I emission intensities yielded values that agreed well with measured values in the near-field plume. However, the significant majority of the data did not yield ratios that were within the ranges acceptable for the models. Often, data next to each other in terms of operating condition yielded very different results. Future efforts to use fiber optic probes to measure $T_{e}$ in the plume will leverage NIR transitions instead of NUV to visible ones. In addition to there being significantly more data regarding collisonalradiative models in that optical regime, the correlations accept a larger range of $\mathrm{T}_{\mathrm{e}}{ }^{20}$

Single-frequency imaging showed detail of the near-field plume which may lend itself to non-intrusive property mapping. Care will have to be taken in the future to align the cameras in such a way that the depth of field is either very short or very deep to allow reasonable interpretation of the data.

\section{Conclusion}

The use of fiber optic probes to characterize the wear and operation of high-power Hall-effect thrusters has been demonstrated. The emission of boron atoms has been normalized using an actinometrical method that mitigates the need to know the electron temperature in the discharge or the detailed excitation and quenching mechanisms of the B I transitions. The technique yielded a real-time, non-intrusive diagnostic that captured trends in BN insulator erosion as various HETs were operated. In this regard, it demonstrated the ability to provide at least a real-time relative assessment of the impact of changes in the operating conditions. Also, the clear differences in $\mathrm{I}_{\mathrm{B}, 250}$ 
collected from probes focused a few mm apart on the BN walls demonstrated the ability to measure with spatial resolutions on the order of a few millimeters within the thruster.

$\mathrm{Au}$ and $\mathrm{Ag}$ foil layers were used to demonstrate the potential of the OES technique incorporating actinometry. While not directly applicable to the life assessment of the thruster itself, the technique did demonstrate the ability to correlate the OES signal with the overall erosion rate of the target materials. Thus, the technique demonstrated the ability to measure $\mathrm{BN}$ erosion rates if those rates can be correlated with $\mathrm{I}_{\mathrm{B}, 250}$.

Insulator erosion trends characterized by the normalized boron neutral atom spectral emission at $250 \mathrm{~nm}$ were obtained on the $3.9 \mathrm{~kW}$ HiVHAc EM thruster, the $20 \mathrm{~kW}$ NASA 300M thruster, and the $50 \mathrm{~kW}$ NASA 457Mv2 thruster. The $N_{B}$ values were linearly proportional to power and $V_{D}$ for the HiVHAc thruster and showed little difference between the inner and outer walls. $\mathrm{N}_{B}$ values for the NASA $300 \mathrm{M}$ thruster showed a greater than linear dependence on power, primarily trending with discharge current. The inner wall of this thruster had much higher $\mathrm{N}_{B}$ values than the outer wall. $\mathrm{N}_{B}$ values of the NASA 457Mv2 thruster also showed an unexpected dependence on $\mathrm{J}_{\mathrm{D}}$ with a slope roughly equal to $\left(1-\eta_{A}\right)$. Why a stronger dependence on $V_{D}$ was not observed for the high-power thrusters remains unclear but may result from the averaging of the Xe lines by the OES probes used to normalize (correct) the raw $\mathrm{I}_{\mathrm{B}, 250}$. More analysis and data are required to confirm these trends or to resolve why the OES technique is not capturing the proper dependence of the erosion rate on operating condition.

Near-field plasma properties were obtained through the ratios of Xe I spectral line intensities. Where these ratios were within the acceptable ranges to fit the models, the values of $\mathrm{T}_{\mathrm{e}}$ agreed well with probe data. However, the use of different transitions will likely greatly enhance the potential applicability of this technique. When coupled with single-frequency imaging, the non-intrusive acquisition of a large amount of near-field data may be possible.

\section{Acknowledgments}

The authors would like to thank and acknowledge the Office of the Chief Technologist for funding this work as well as Timothy Smith for serving as the Project Manager. The authors also thank Kevin McCormick for his aid in fabricating the metallic coupons used in this study and George Readus, George Jacynycz and Kevin Blake for maintaining and operating the vacuum facilities.

\section{References}

${ }^{1}$ Dudzinski, L., et al., "Design of Solar Electric Propulsion Transfer Vehicle for a Non-Nuclear Human Mars Exploration Architecture," 26th International Electric Propulsion Conference Paper IEPC-99-181, Japan, October 1999.

2 Oleson, S.R., “Advanced Propulsion for Space Solar Power Satellites," $35^{\text {th }}$ AIAA Joint Propulsion Conference, AIAA-992872, Los Angeles, California, June 1999.

${ }^{3}$ Oleson, S.R., et al., "Mission Advantages of Constant Power Variable Specific Impulse Electrostatic Thrusters," NASA TM-2000-210477, 2000.

${ }^{4}$ Leray, P., et al., "Spatially Resolved Emission Spectroscopy Along a SPT Channel, Interpretation of Data by a CollisionalRadiative Model,” International Electric Propulsion Conference Paper IEPC-97-054, Cleveland, Ohio 1997

${ }^{5}$ Karabadzhak, G. F., and Semenkin, A. V., "Investigation of TAL Optical Emissions," International Electric Propulsion Conference Paper IEPC-97-131, Cleveland, Ohio, 1997.

${ }^{6}$ Pagnon, D., Touzeau, M., and Lasgorceix, P., "Control of the Ceramic Erosion by Optical Emission Spectroscopy: Parametric Studies of SPT 100-ML,” AIAA Paper2004-3773, July 2004.

${ }^{7}$ Hargus, W. A., "Optical Boron Nitride Insulator erosion Characterization of a $200 \mathrm{~W}$ Xenon Hall Thruster," 41st AIAA Joint Propulsion Conference, AIAA-2005-3529, Tucson, Arizona, 2005.

${ }^{8}$ Cho, S., et al., "Experimental and Numerical Study on a Hall Thruster Insulator Erosion," International Electric Propulsion Conference Paper IEPC-2011-078, Wiesbaden, Germany, September 2011.

${ }^{9}$ Shmelev, A. V., and Lovtsov, A., S., "Investigation of Discharge Power Influence on Erosion Rate of SPT Discharge Chamber Using Spectroscopic Method," International Electric Propulsion Conference Paper IEPC-2011-024, Wiesbaden, Germany, September 2011 
${ }^{10}$ Kozintseva, M. V., et al., "Forecast of Erosion Rate of SPT K-0 Channel Insulators by Spectral Measurements," International Electric Propulsion Conference Paper IEPC-2011-293, Wiesbaden, Germany, September 2011.

${ }^{11}$ Cho, S., et al., "Hall Thruster Channel Wall Erosion Rate Measurement Method Using Multilayer Coating Chip," $46^{\text {th }}$ AIAA Joint Propulsion Conference, AIAA-2010-6697, Nashville, Tennessee, July 2010.

12 Arabadzhak, G. F. and Semenkin, A. V., "Evaluation of a Xenon Operating Hall Thruster Body Erosion Rate Through Analysis of Its Optical Spectra,” $37^{\text {th }}$ AIAA Joint Propulsion Conference, AIAA-2001-3889, Salt Lake City, Utah, July 2001.

${ }^{13}$ Manzella, D. H., et al., "Laboratory Model 50 kW Hall Thruster," $38^{\text {th }}$ AIAA Joint Propulsion Conference, AIAA-2002-3676, Indianapolis, Indiana, July 2002.

${ }^{14}$ Dunning, J. and Sankovic, J., "NASA's Electric Propulsion Program," $36^{\text {th }}$ AIAA Joint Propulsion Conference AIAA Paper 2000-3145, Huntsville, Alabama, July 2000.

${ }^{15}$ Kamhawi, Hani, et al., "Overview of Hall Thruster Activities at NASA Glenn Research Center," International Electric Propulsion conference Paper IEPC-2011-339, Wiesbaden, Germany, 2011.

16 Herman, D. A., Shastry, R., Huang, W., Soulas, G. C., and Kamhawi, H., "Plasma Potential and Langmuir Probe Measurements in the Near-field Plume of the NASA 300M Hall Thruster," Presented at the 48th AIAA/ASME/SAE/ASEE Joint Propulsion Conference and Exhibit, Atlanta, GA, July 29 - August 1, 2012.

${ }^{17}$ Soulas, G. C., Haag, T. W., Herman, D. A., Huang, W., Kamhawi, H. et al., "Performance Test Results of the NASA-457M v2 Hall Thruster," 48 ${ }^{\text {th }}$ AIAA/ASME/SAE/ASEE Joint Propulsion Conference and Exhibit, AIAA-2012-3940, Atlanta, GA, July 29 - August 1, 2012.

${ }^{18}$ Oh, D. "Evaluation of Solar Electric Propulsion Technologies for Discovery Class Missions," 41 st AIAA Joint Propulsion Conference, AIAA-2005-4270, Tucson, Arizona, July 2005.

${ }^{19}$ Manzella, D., Oh, D., and Aadland, R., "Hall Thruster Technology for NASA Science Missions," 41st AIAA Joint Propulsion Conference, AIAA-2005-3675, Tucson, Arizona, 2005.

${ }^{20}$ Chiu, Y. H., et al., "Passive Optical Diagnostic of Xe Propelled Hall Thrusters, Part 1: Emission Cross Sections

${ }^{21}$ Selwyn, G. S., Optical Diagnostic Techniques for Plasma Processing, American Vacuum Society, New York, 1993, pp47-50.

${ }^{22}$ Balance, C. P., Griffin, D. C., Berrington, K. A., and Badnell, N. R., "Electron-impact Excitation of Neutral Boron Using the R-matrix with Pseudostates Method," Journal of Physics B: Atomic, Molecular and Optical Physics, 40 (2007) 1131-1139.

${ }^{23}$ Lotz, W., "Electron-Impact Ionization Cross-sections and Ionization Rate Coefficients for Atoms and Ions," ApJS, 14, 207L, 1967.

${ }^{24}$ Nelson, A. N., "Electron Impact Ionization Cross-Sections of God, Chromium and Iron,” AFML Technical Report AFML-TR75-198, December, 1973.

${ }^{25}$ Franzreh, K., Wucher, A., and Oechsner, H, “Absolute Cross-sections for Electron Impact Ionization of Ag2," Z.Phys D Atoms, Molecules and Clusters 19, 77-79, 1991.

${ }^{26}$ Fowles, G. R., Modern Optics, $2^{\text {nd }}$ ed., Dover Publications, New York, 1968, pp 294-304.

27 Jameson, K.K., Goebel, D. M., and Watkins, R. M., "Neutral Density Measurements in a Hall Thruster Plume," $43^{\text {rd }}$ AIAA Joint Propulsion Conference, AIAA-2007-5853, Cincinnati, Ohio, July, 2007.

${ }^{28}$ Haas, J. M., Hofer, R. R., and Gallimore, A. D., "Hall Thruster Discharge Chamber Plasma Characterization Using a HighSpeed Axial Reciprocating Electrostatic Probe," 38 th AIAA Joint Propulsion Conference, AIAA-99-2426, Los Angeles, California, June, 1999. 
Table 1 Comparison of Properties of Sputtered Species (Optical properties of B are given for BN)

\begin{tabular}{|c|c|c|c|c|c|}
\hline \multirow{2}{*}{ Material } & \multicolumn{3}{|c|}{ Volumetric sputter yield, cc/mol } & \multirow{2}{*}{$\begin{array}{l}\text { Wavelength of } \\
\text { interrogation, } \mathrm{nm}\end{array}$} & \multirow{2}{*}{$\begin{array}{c}\text { Energy of } \\
\text { upper state, } \mathrm{eV}\end{array}$} \\
\hline & $100 \mathrm{eV}$ & $200 \mathrm{eV}$ & $300 \mathrm{eV}$ & & \\
\hline$\overline{\mathrm{BN}}(\mathrm{B})$ & 0.48 & 1.54 & 1.93 & 249.8 & 3.45 \\
\hline $\mathrm{Au}$ & 1.63 & 10.2 & 18.7 & 242.8 & 3.55 \\
\hline $\mathrm{Ag}$ & 4.11 & 10.8 & 18.5 & 328.1 & 2.63 \\
\hline
\end{tabular}

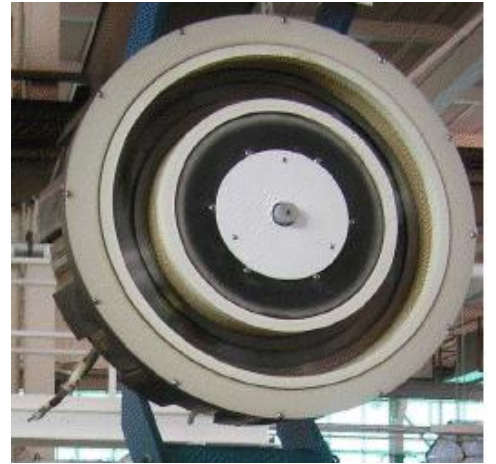

a. NASA $457 \mathrm{Mv} 2$

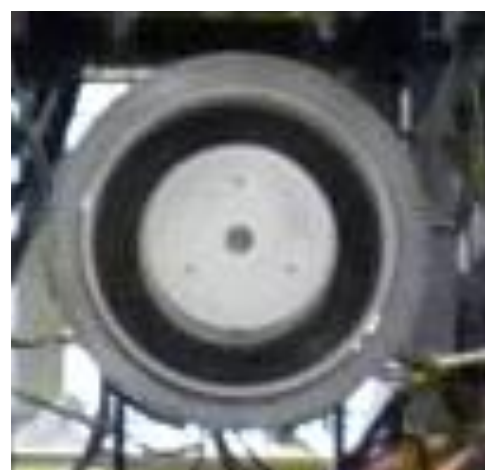

b. NASA 300M

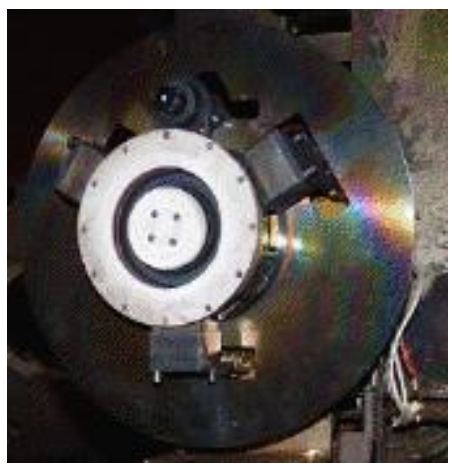

c. HiVHAc EM

Figure 1 Photographs of the Hall effect thrusters used in this investigation. ${ }^{15}$

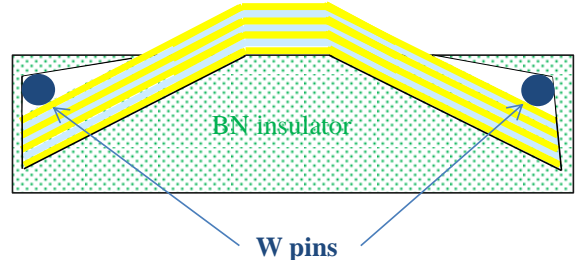

a. Schematic of the coupon layers/

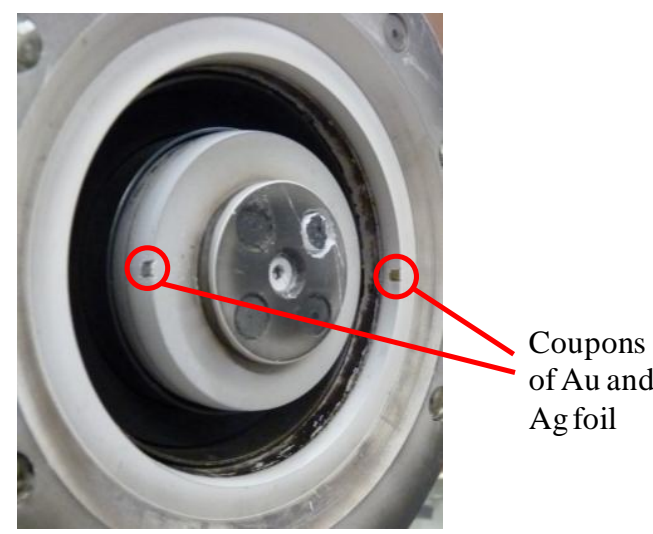

b. Photograph of the coupons on the HiVHAc thruster.

Figure 2 Coupon configurations.

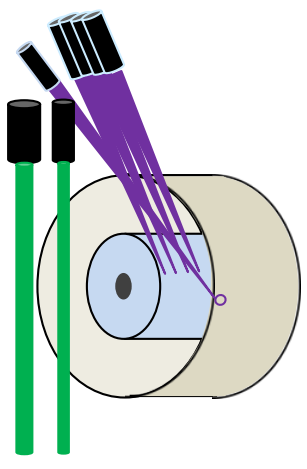

a. NASA 457Mv2

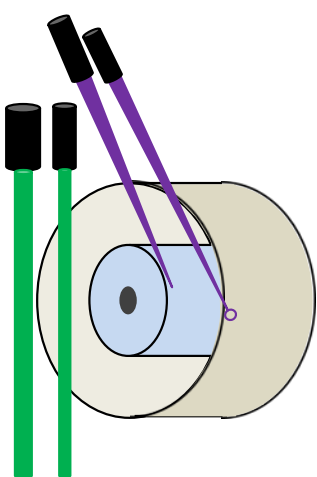

b. NASA 300M

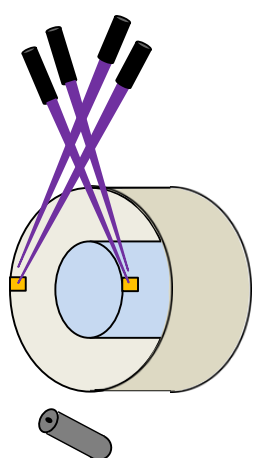

c. HiVHAc EM

Figure 3 Schematic alignment of the optical probes used to interrogate the three thrusters. Drawings are not to scale. 


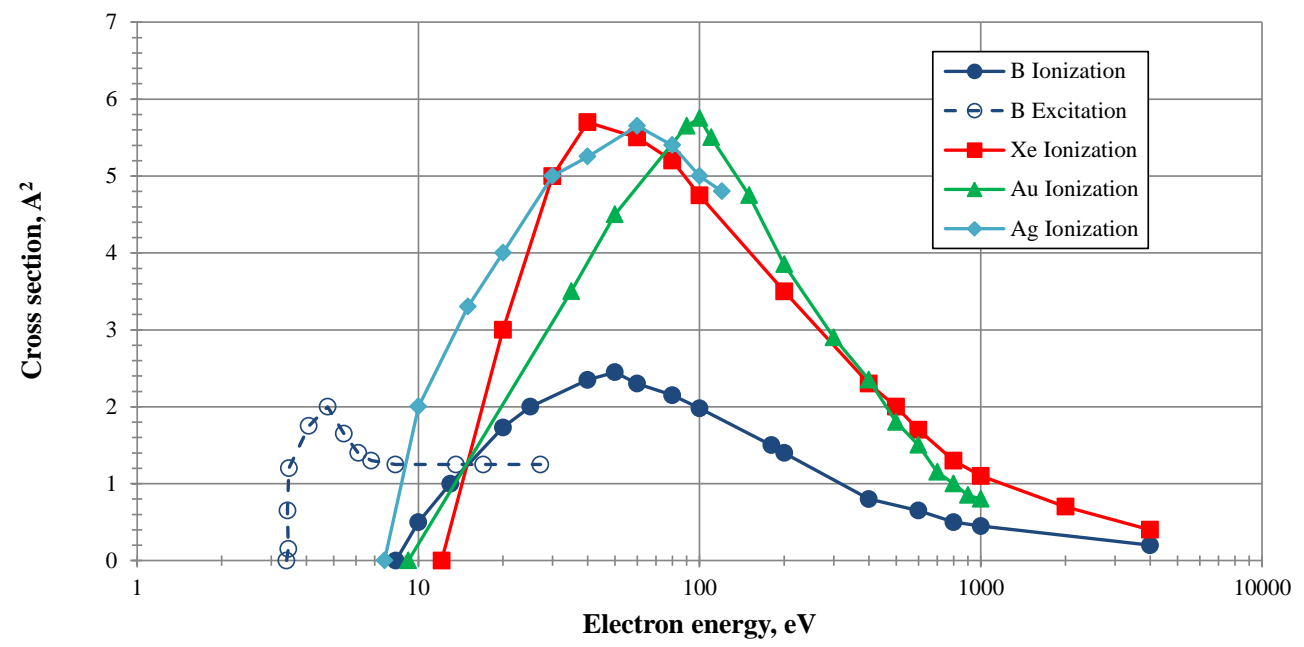

Figure 4 Electron-impact ionization cross-sections for $\mathrm{B}, \mathrm{Au}, \mathrm{Ag}$, and $\mathrm{Xe}$ and the electron impact excitation cross-section for the $250 \mathrm{~nm}$ B I transition as a function of electron energy.

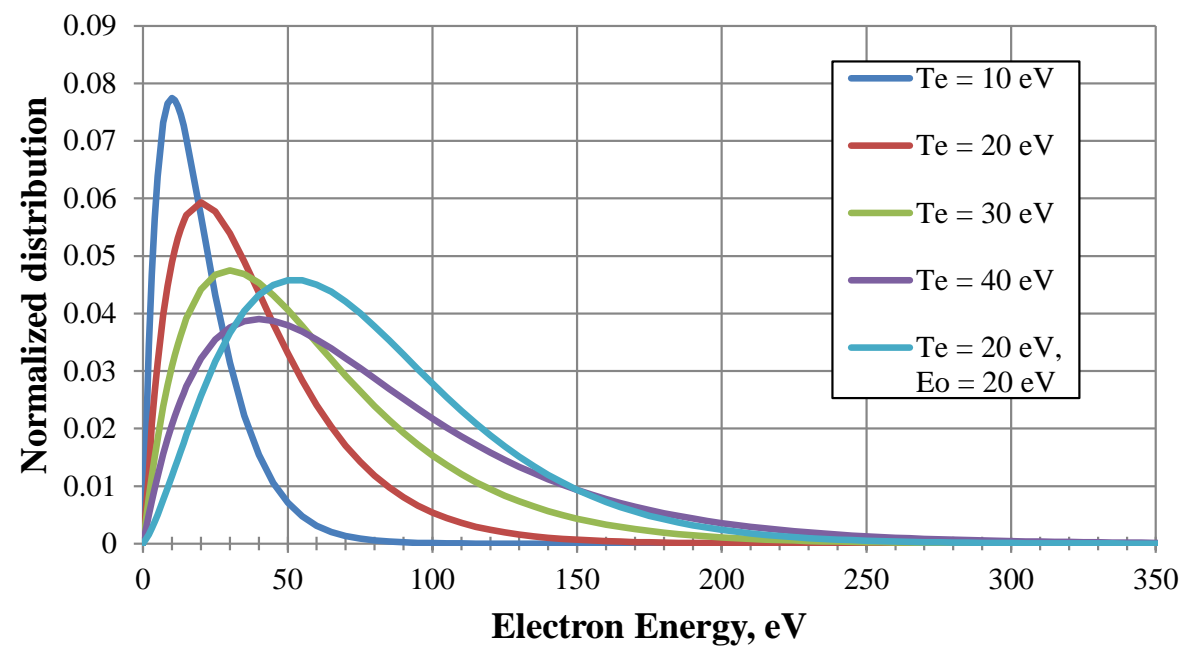

Figure 5 Maxwellian electron energy distributions for a number of electron temperatures and the distribution of electrons for a moving Maxwellian with bulk energy and temperature equal to $20 \mathrm{eV}$. Each distribution is normalized by its integrated value.

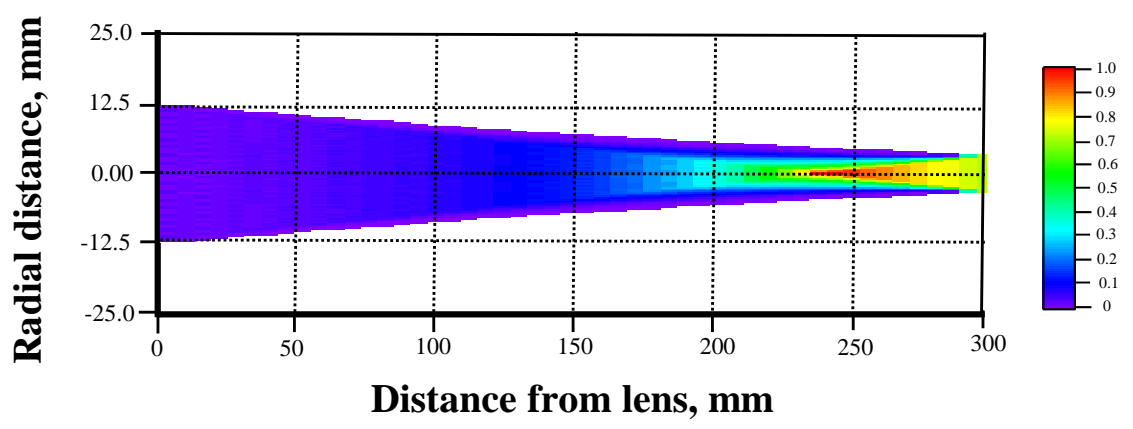

Figure 6 Calculated fraction of light collection within the cone of interrogation of the emissive probes. The fraction is normalized to its maximum. The relative scale of the axes is shown in Fig. 7. 


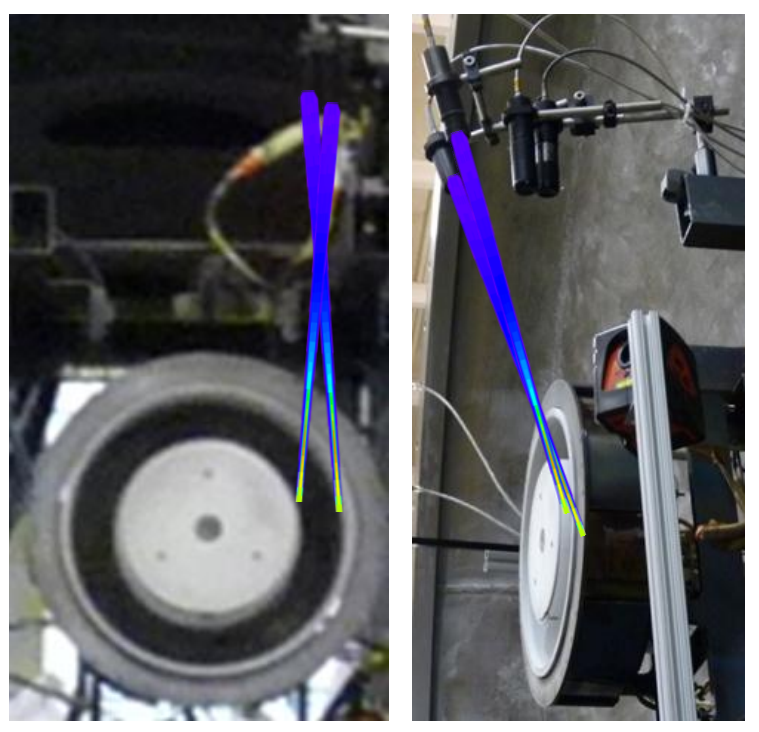

Figure 7 Superposition of the sampling volume shown in Fig. 6 onto the test configuration of the NASA 300M. Note that the regions of relatively high sampling are in the plume and the regions of lower sampling are largely outside of it.

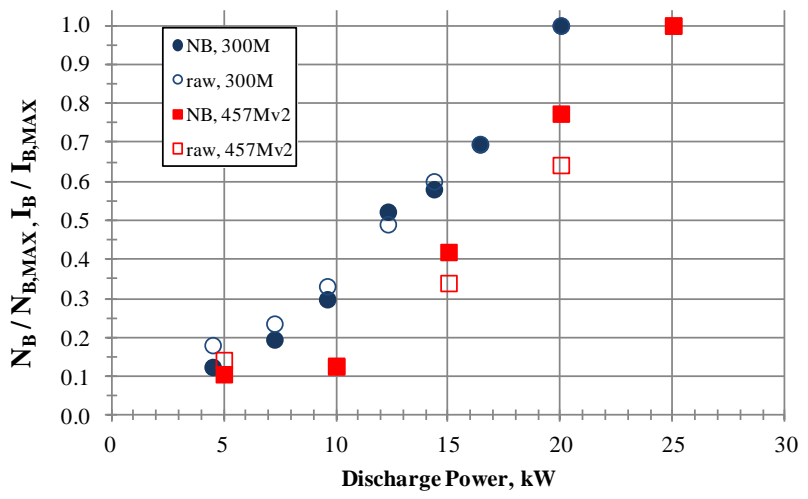

a. $\mathrm{V}_{\mathrm{D}}=250 \mathrm{~V}$

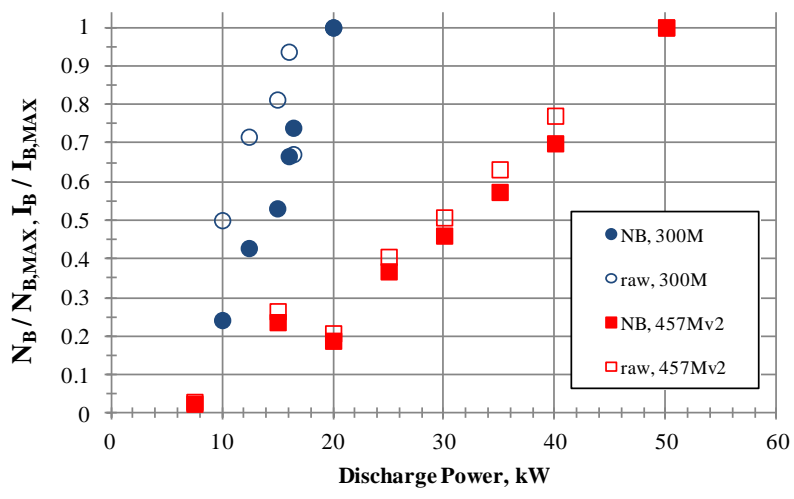

b. $\mathrm{V}_{\mathrm{D}}=400 \mathrm{~V}$

Figure 8 Comparison of $\mathrm{N}_{\mathrm{B}}$ and $\mathrm{I}_{\mathrm{B}, 250}$ values from the NASA 300M and NASA 457Mv2 as a function of discharge power. Since the signals are normalized separately for each thruster, only the trends and not the relative values can be compared.

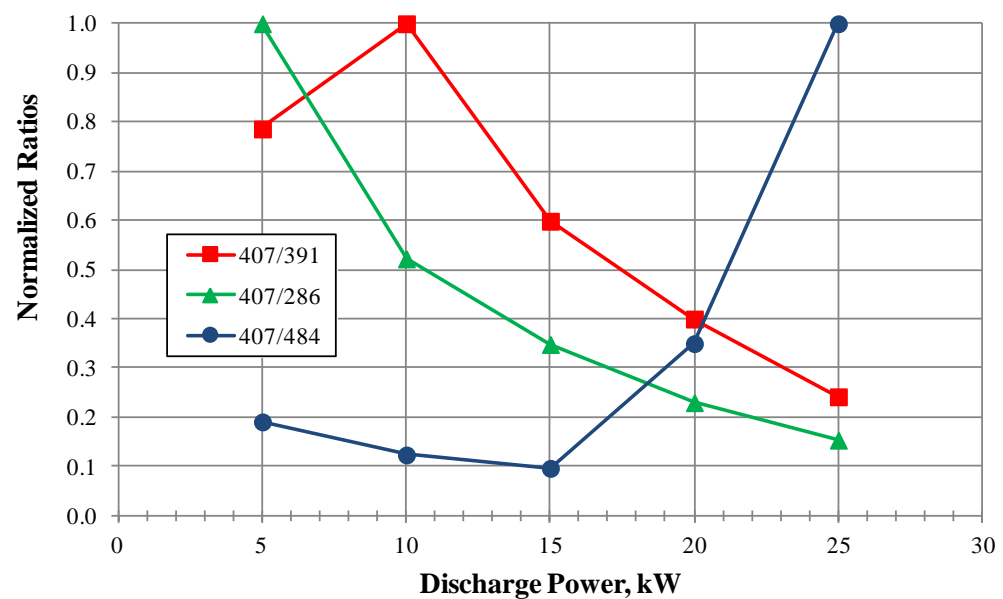

Figure 9 Comparison of trends of the ratio of the intensity of the Xe I $407 \mathrm{~nm}$ line with intensities of three Xe II lines: $286 \mathrm{~nm}, 391, \mathrm{~nm}$ and $484 \mathrm{~nm}$. Data are taken from the discharge of the NASA 457Mv2 thruster. 


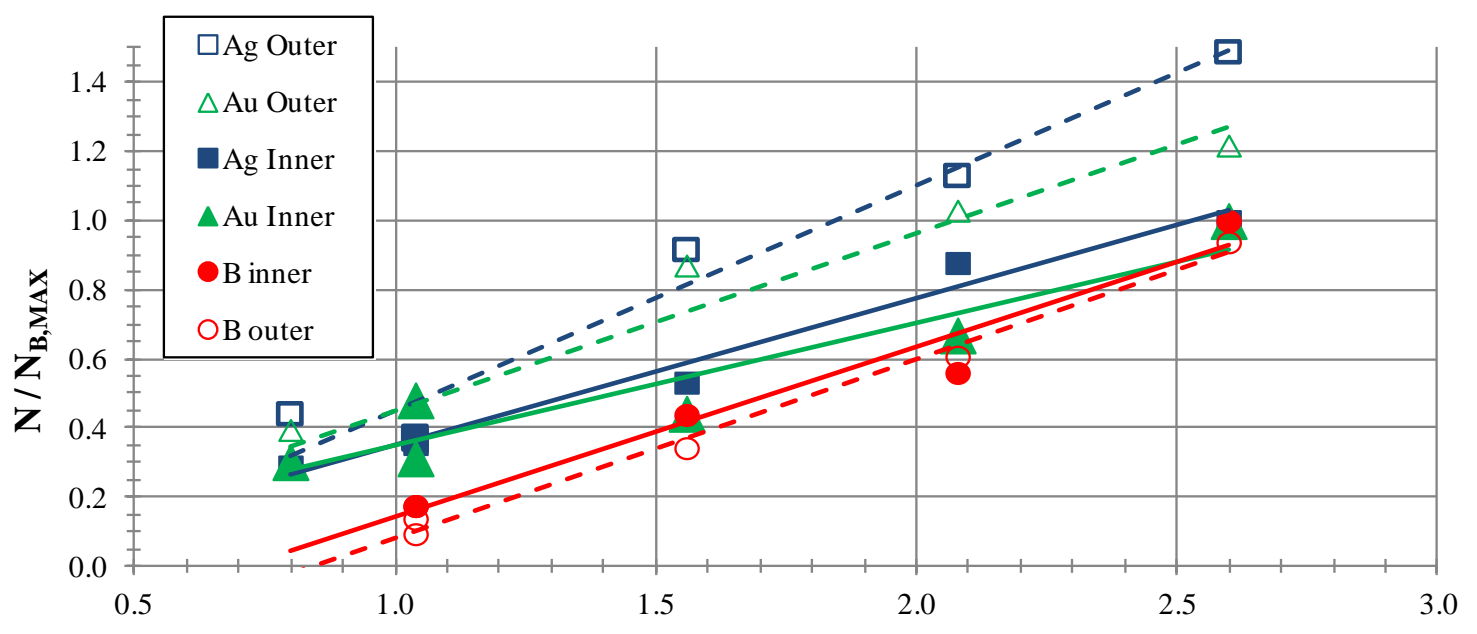

Discharge Power, kW

Figure 10 Comparison of $\mathrm{Au} \mathrm{I,} \mathrm{Ag} \mathrm{I,} \mathrm{and} \mathrm{B} \mathrm{I} \mathrm{signals} \mathrm{collected} \mathrm{from} \mathrm{the} \mathrm{inner} \mathrm{and} \mathrm{outer} \mathrm{wall} \mathrm{areas} \mathrm{of}$ the HiVHAc EM thruster. Linear trend lines are for ease of reading only.

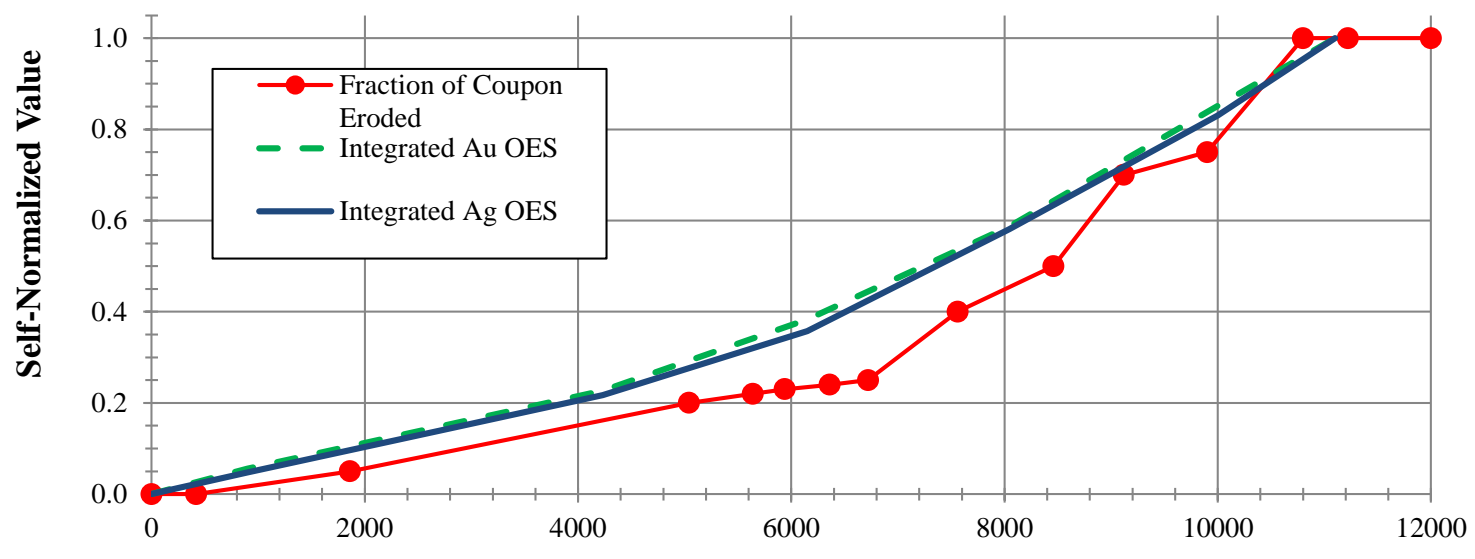

Time, $\mathrm{s}$

Figure 11 Comparison of observed coupon erosion and integrated Au I $247 \mathrm{~nm}$ and Ag I $328 \mathrm{~nm}$ OES signals from the outer wall of the HiVHAc EM thruster.

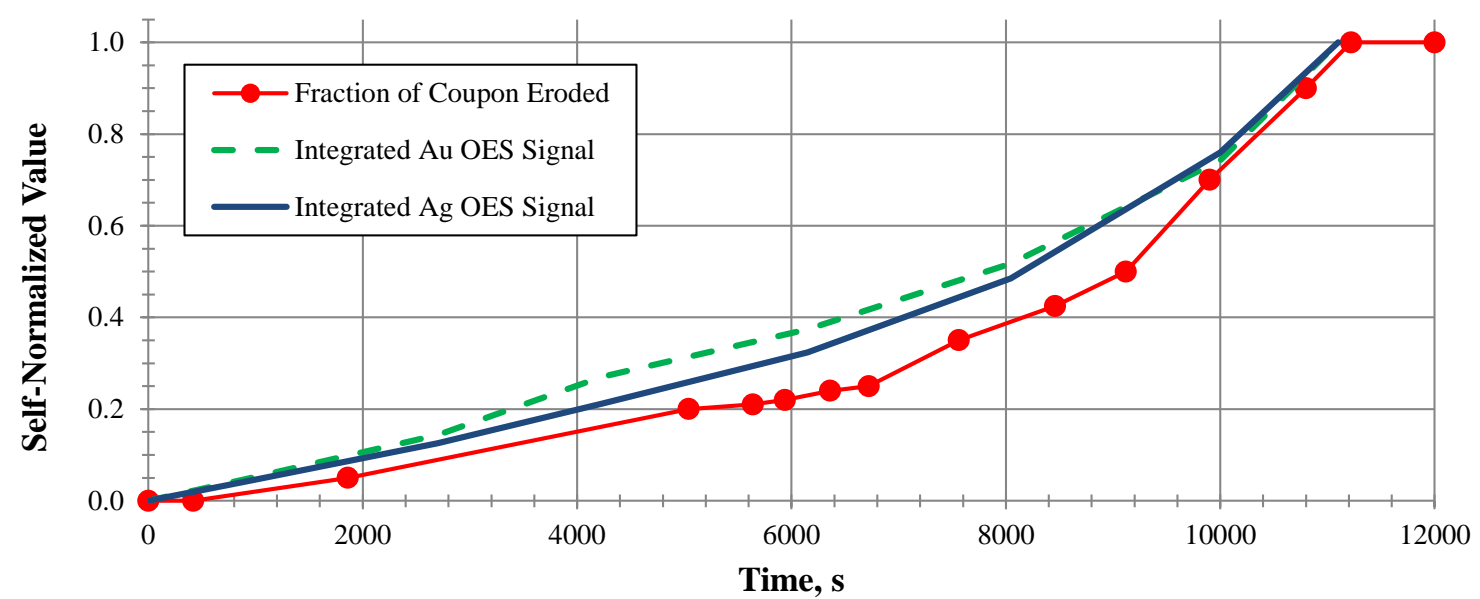

Figure 12 Comparison of observed coupon erosion and integrated Au I $247 \mathrm{~nm}$ and Ag I $328 \mathrm{~nm}$ OES signals from the inner wall of the HiVHAc EM thruster. 


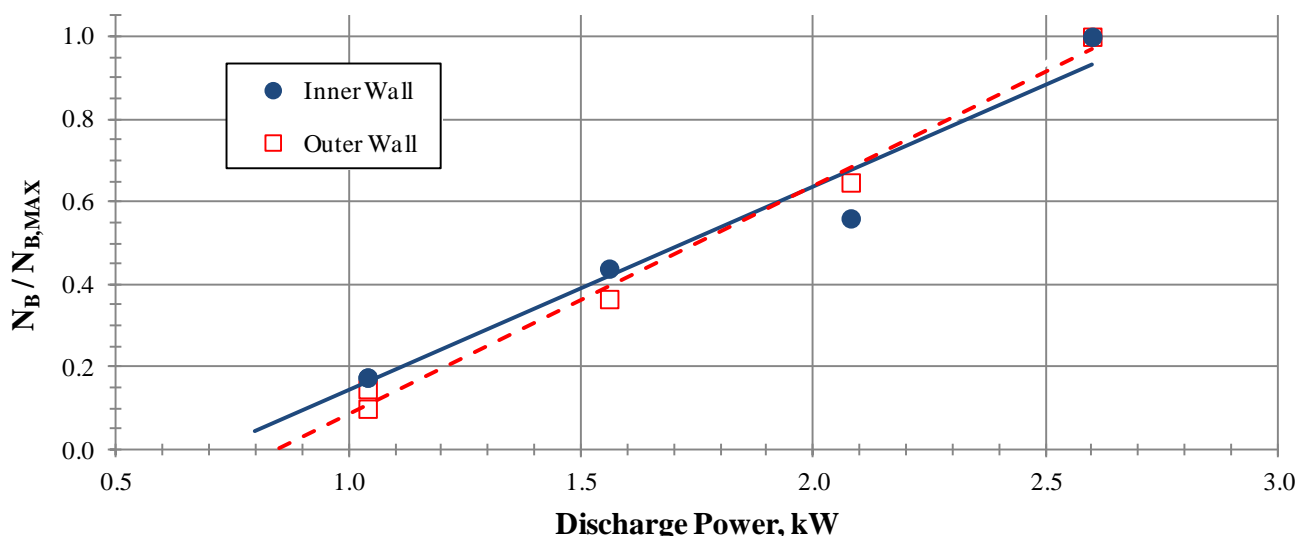

a. HiVHAc EM thruster operating at $\mathrm{J}_{\mathrm{D}}=5 \mathrm{~A}$

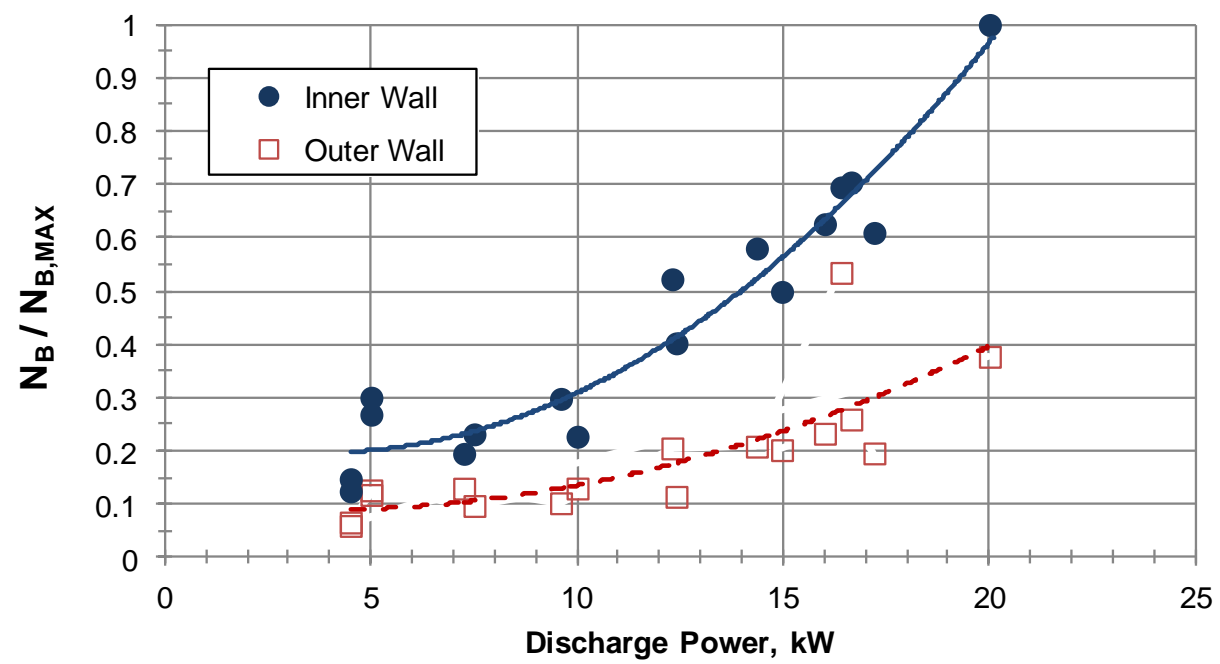

b. NASA 300M ER thruster operating at $500 \mathrm{~V}>\mathrm{V}_{\mathrm{D}}>200 \mathrm{~V}$.

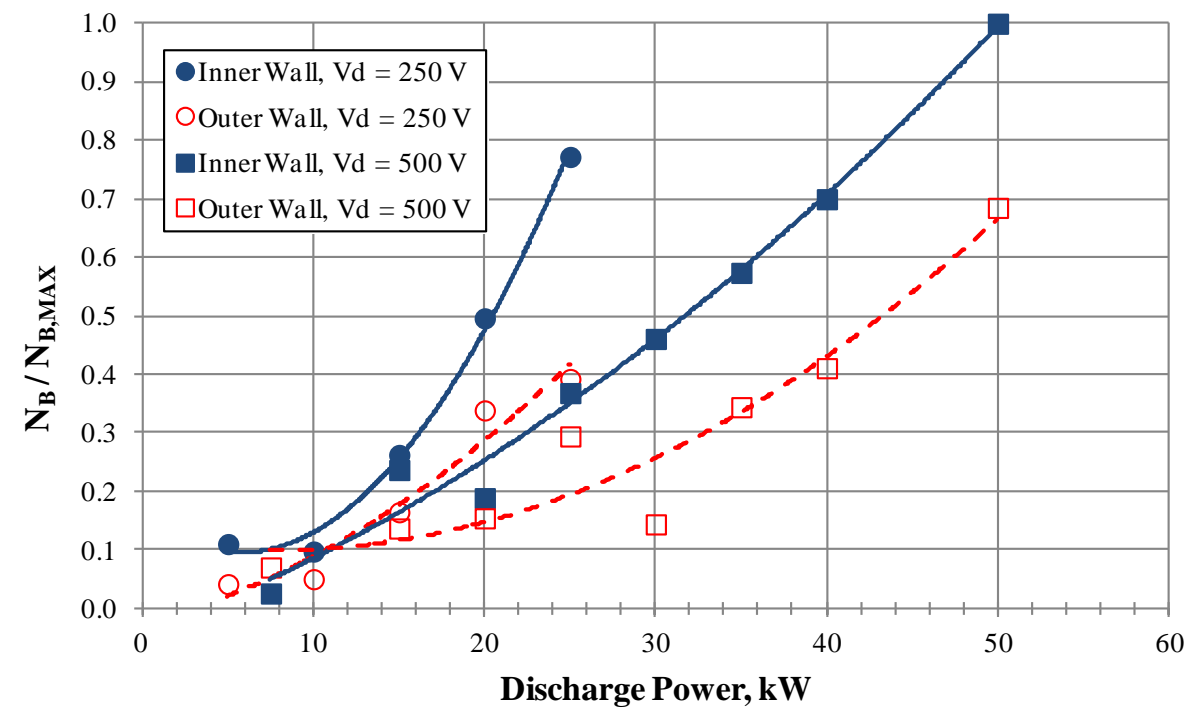

c. NASA 457Mv2 thruster operating at discharge voltages of $250 \mathrm{~V}$ and $500 \mathrm{~V}$.

Figure 13 Comparison of B OES signals from the inner and outer walls as a function of discharge power for three HETs. All data are normalized to the peak value of the inner wall's signal within a given plot

American Institute of Aeronautics and Astronautics 


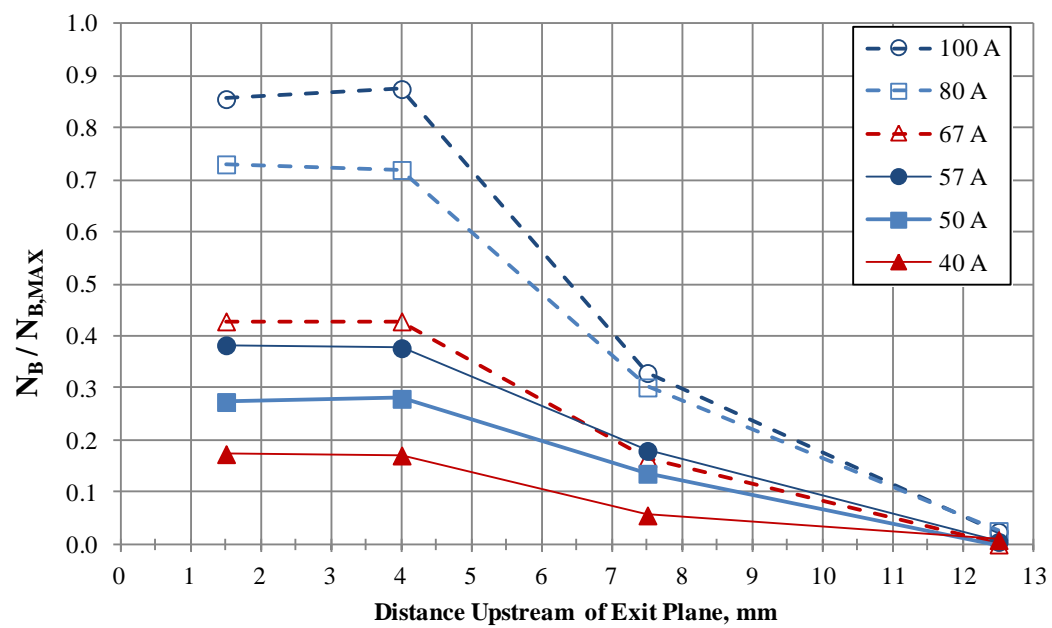

Figure 14 Trends in B OES signal as a function of axial location for $20 \mathrm{~kW}$ operation of the NASA $457 \mathrm{Mv} 2$ thruster.

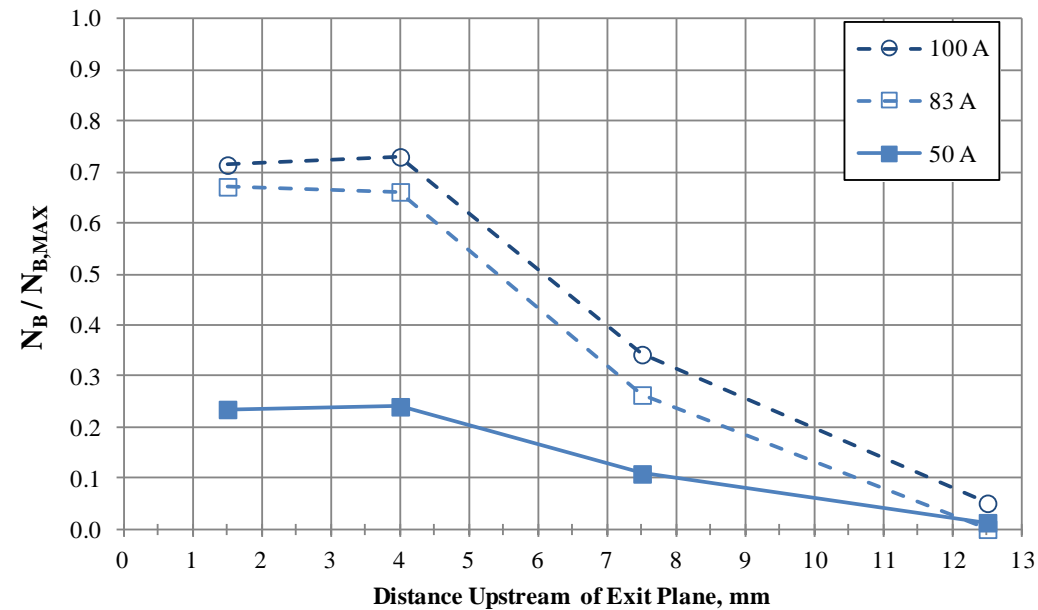

Figure 15 Trends in B OES signal as a function of axial location for $25 \mathrm{~kW}$ operation of the NASA $457 \mathrm{Mv} 2$ thruster.

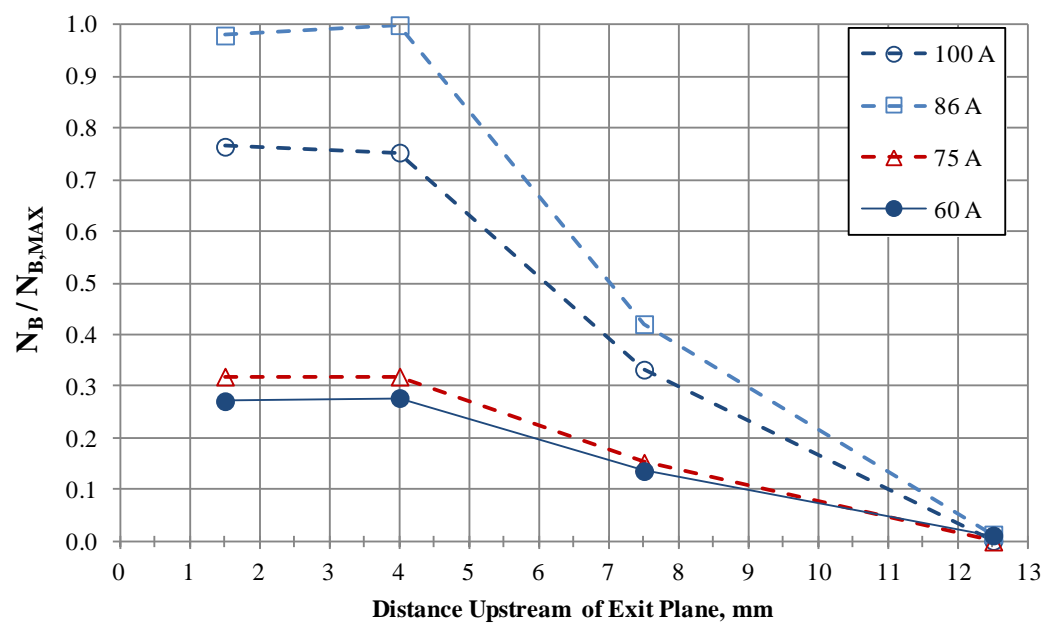

Figure 16 Trends in B OES signal as a function of axial location for $30 \mathrm{~kW}$ operation of the NASA $457 \mathrm{Mv} 2$ thruster. 


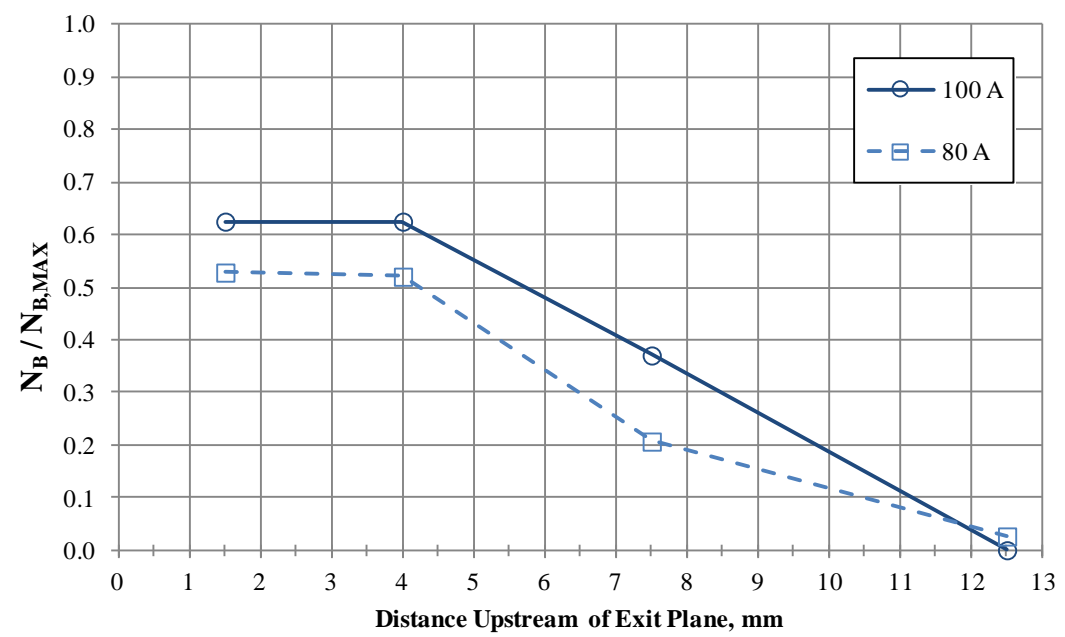

Figure 17 Trends in B OES signal as a function of axial location for $40 \mathrm{~kW}$ operation of the NASA $457 \mathrm{Mv} 2$ thruster.

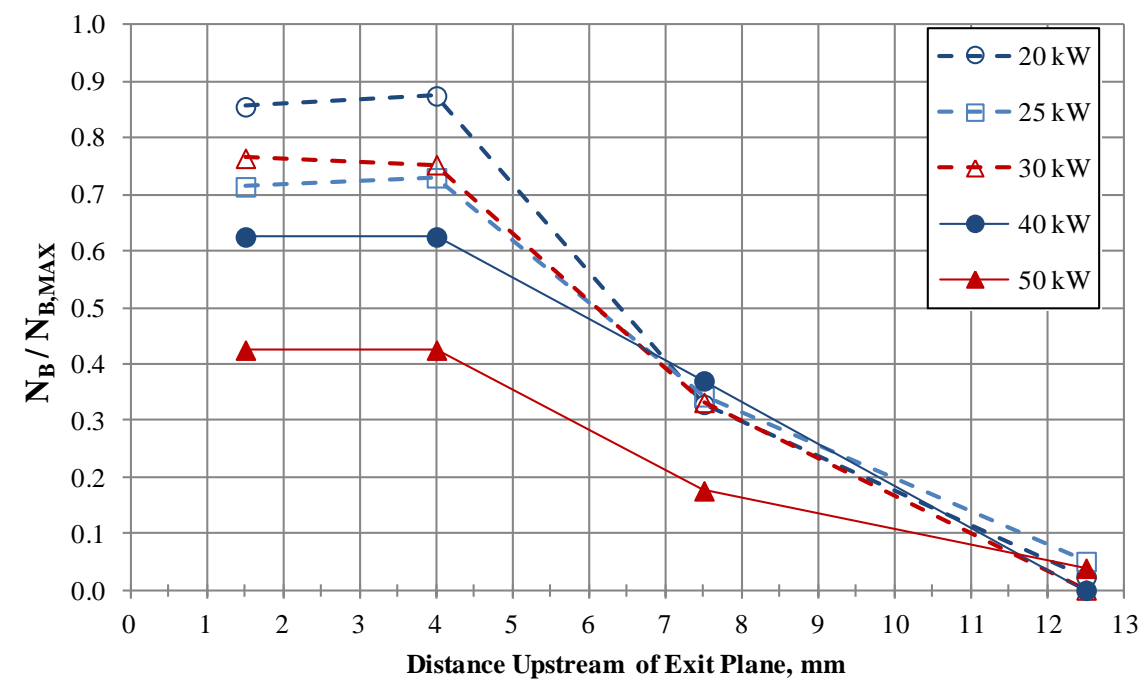

Figure 18 Trends in $\mathrm{N}_{\mathrm{B}}$ values as a function of axial location for $100 \mathrm{~A}$ operation of the NASA 457Mv2 thruster.

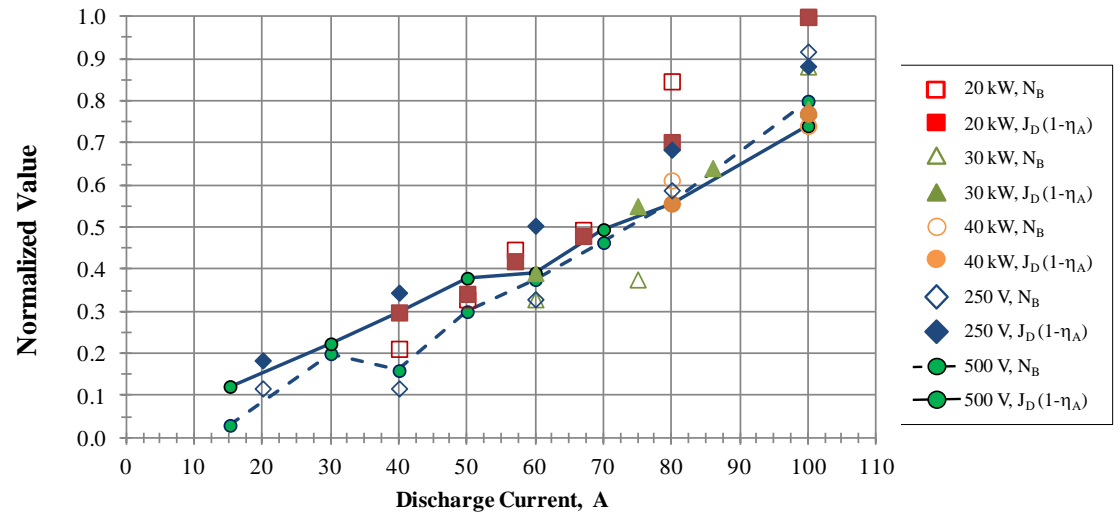

Figure 19 Comparison of normalized $N_{B}$ values and $J_{D}\left(1-\eta_{A}\right)$ values ${ }^{17}$ for the NASA 457Mv2 as a function of $J_{D}$ over a range of operating conditions. As in Figs. 14-18, $N_{B}$ is normalized by the maximum $N_{B}$ in the data plotted and $J_{D}\left(1-\eta_{A}\right)$ is normalized by the maximum $\mathrm{J}_{\mathrm{D}}\left(1-\eta_{\mathrm{A}}\right)$ in the data plotted. 


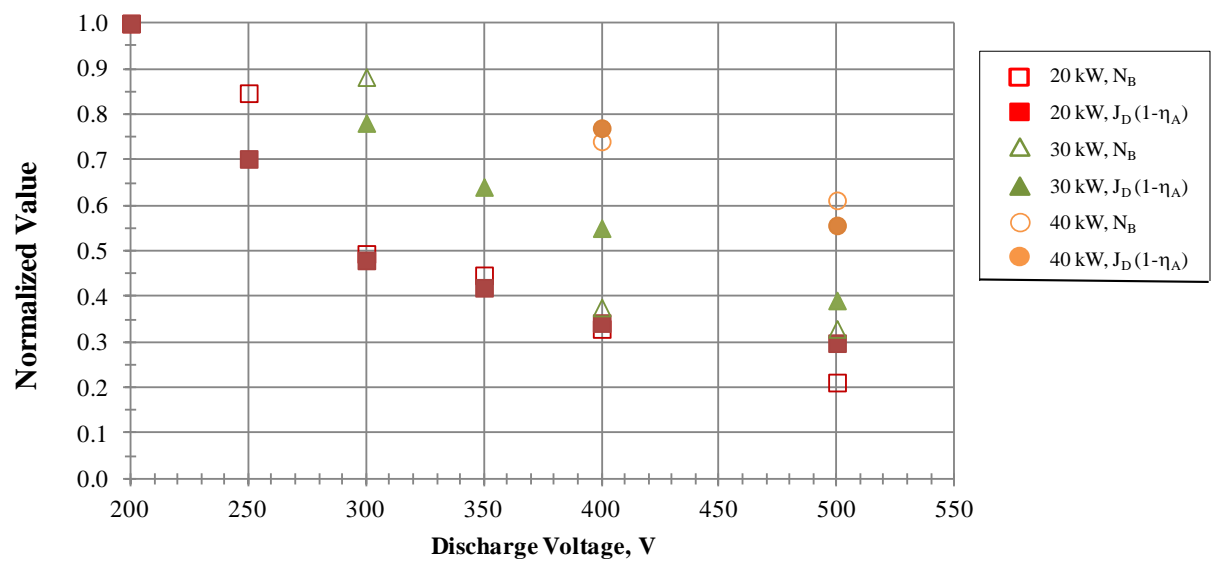

Figure 20 Comparison of normalized $N_{B}$ values and $J_{D}\left(1-\eta_{A}\right)$ values ${ }^{17}$ for the NASA 457Mv2 as a function of $V_{D}$ over a range of operating conditions.

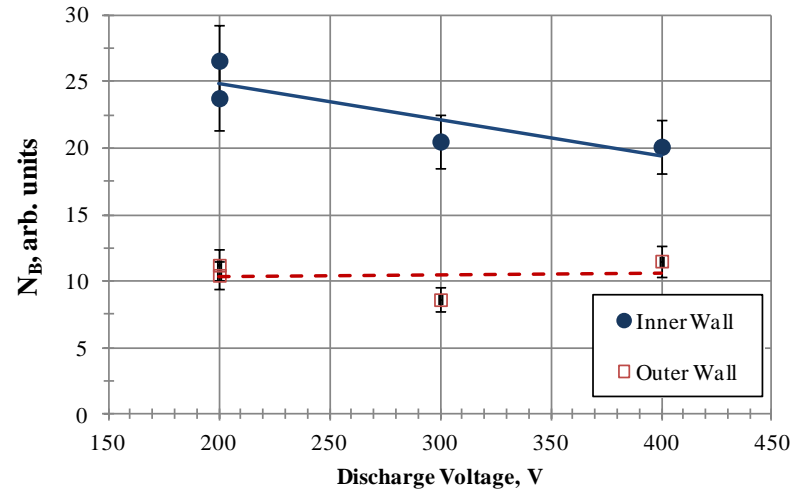

a. $J_{D}=25 \mathrm{~A}$

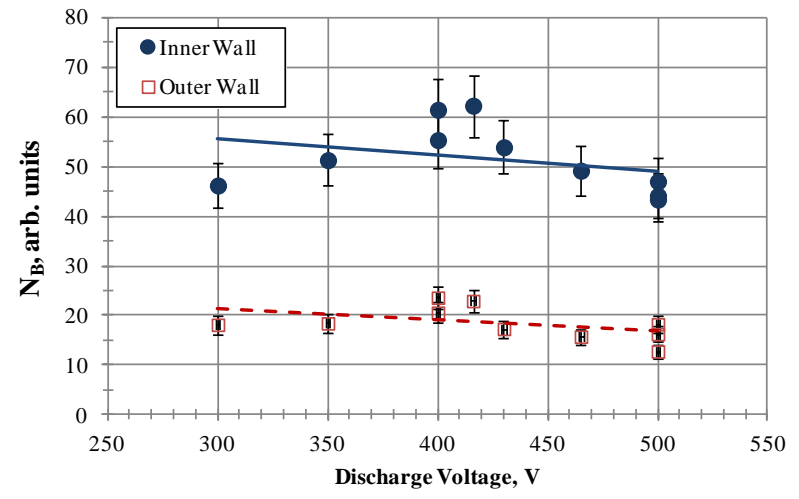

b. $\mathrm{J}_{\mathrm{D}}=40 \mathrm{~A}$

Figure $21 \mathrm{~N}_{\mathrm{B}}$ values as a function of discharge voltage for the inner and outer walls of the NASA 300M thruster.

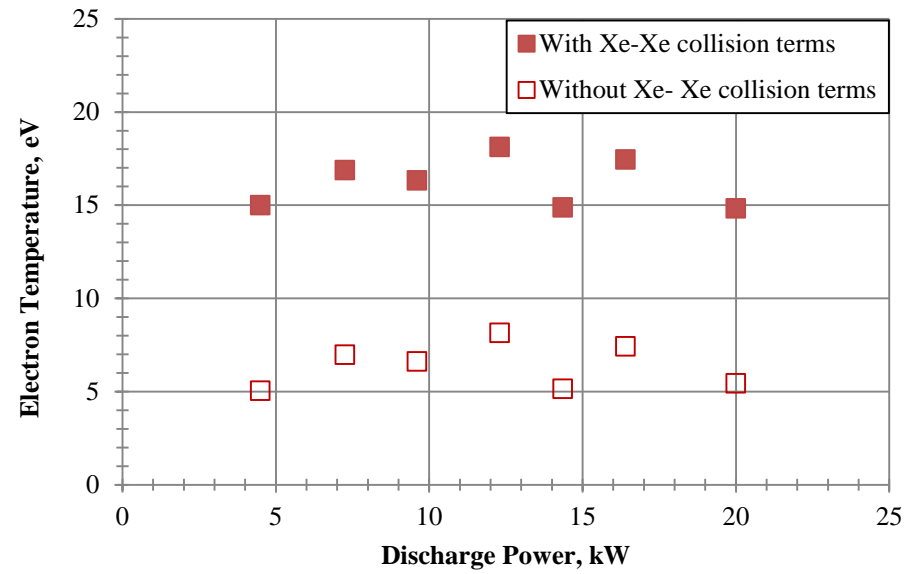

Figure 22 Comparison of near-field electron temperatures measured $15 \mathrm{~cm}$ downstream of the NASA $300 \mathrm{M}$ thruster. 


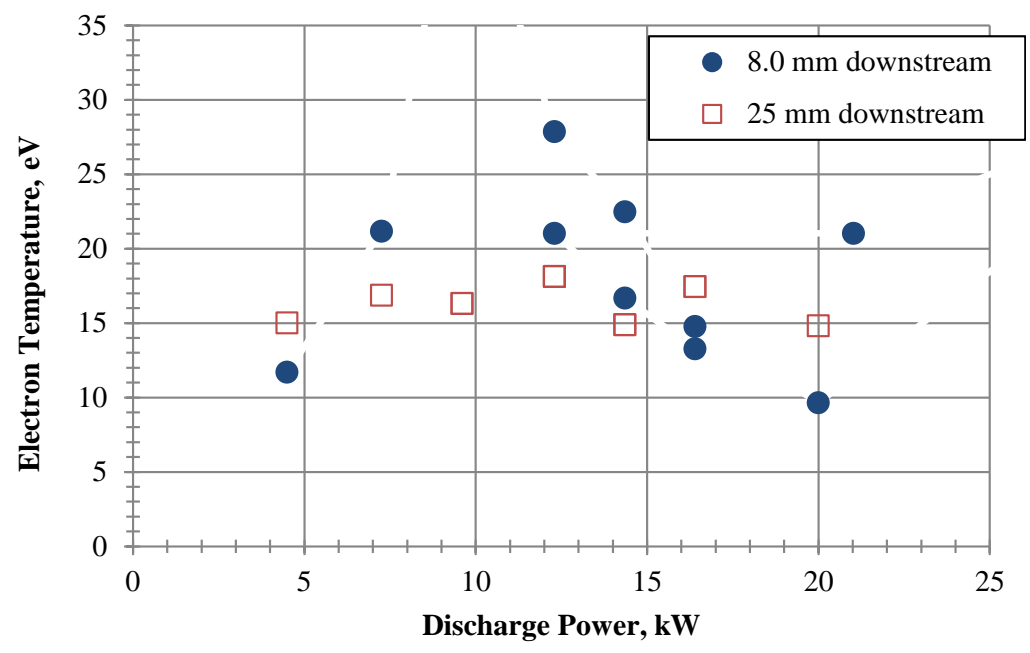

Figure 23 Electron temperatures in the near-field plume of the NASA 300M calculated using the ratio of Xe I $462 \mathrm{~nm}$ and Xe I $473 \mathrm{~nm}$ OES signals.

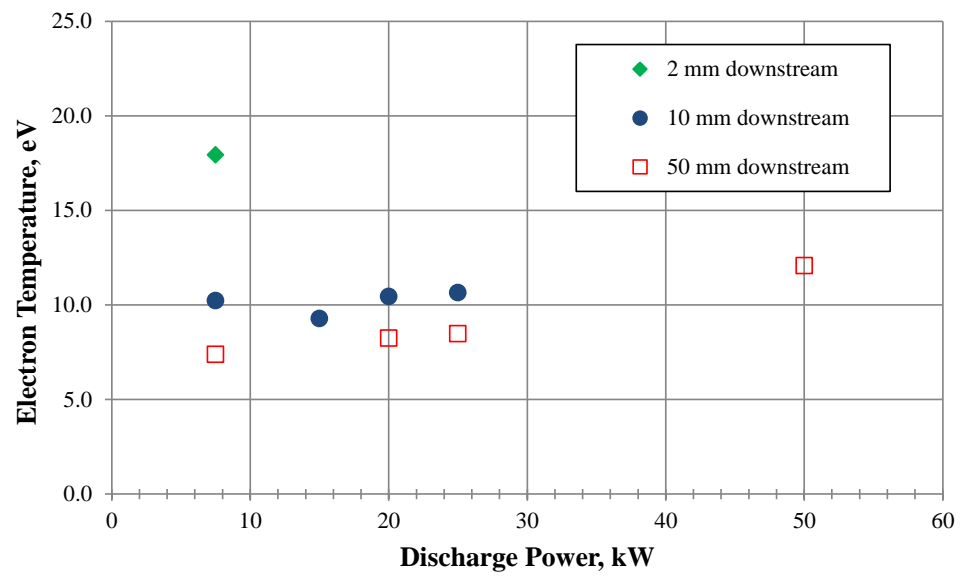

Figure 24 Electron temperatures in the near-field plume of the NASA $457 \mathrm{Mv} 2$ operating at $V_{D}=500 \mathrm{~V}$. $\mathrm{T}_{\mathrm{e}}$ was calculated using the ratio of Xe I $462 \mathrm{~nm}$ and Xe I $473 \mathrm{~nm}$ OES signals.

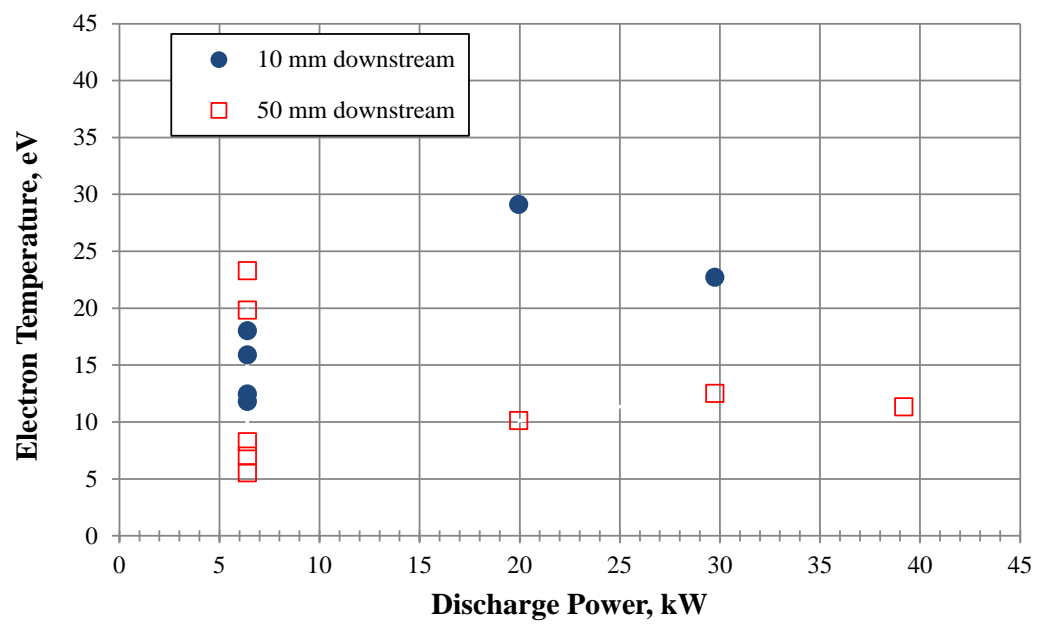

Figure 25 Electron temperatures in the near-field plume of the NASA $457 \mathrm{Mv} 2$ operating at $V_{D}=400 \mathrm{~V}$. $T_{e}$ was calculated using the ratio of Xe I $462 \mathrm{~nm}$ and Xe I $473 \mathrm{~nm}$ OES signals

American Institute of Aeronautics and Astronautics 


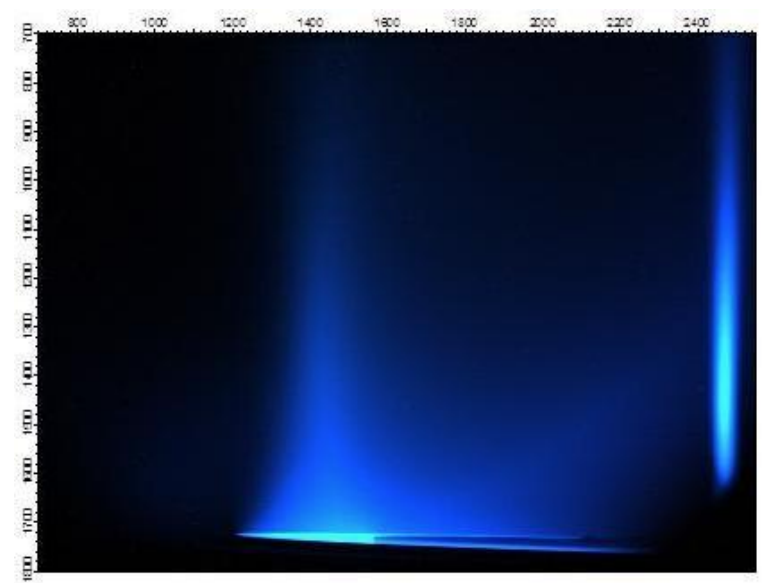

a. Xe I imaged at $473 \mathrm{~nm}$

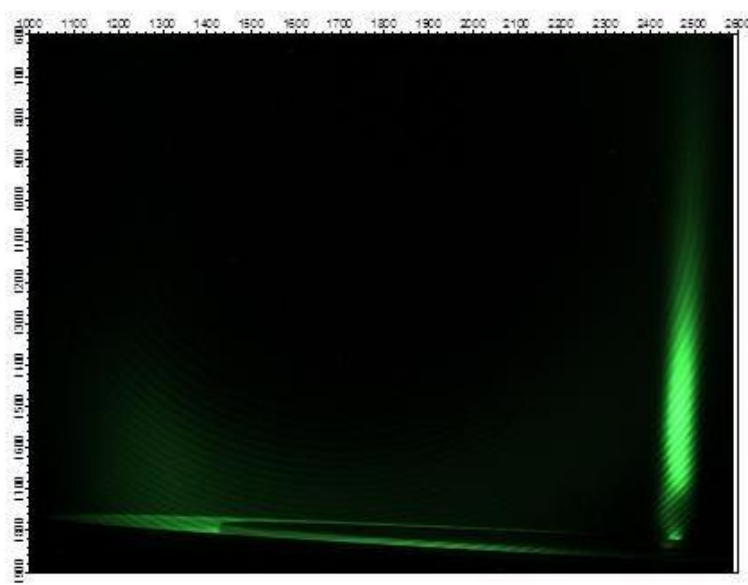

b. Xe II imaged at $541 \mathrm{~nm}$

Figure 26 Photographs of the near-field plume of the NASA 457Mv2 operating at $V_{D}=400 \mathrm{~V}$ and $J_{D}=100 \mathrm{~A}$.

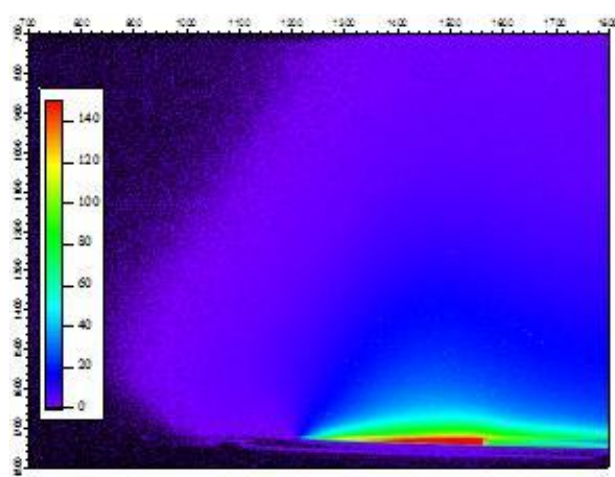

a. $\mathrm{J}_{\mathrm{D}}=16 \mathrm{~A}$

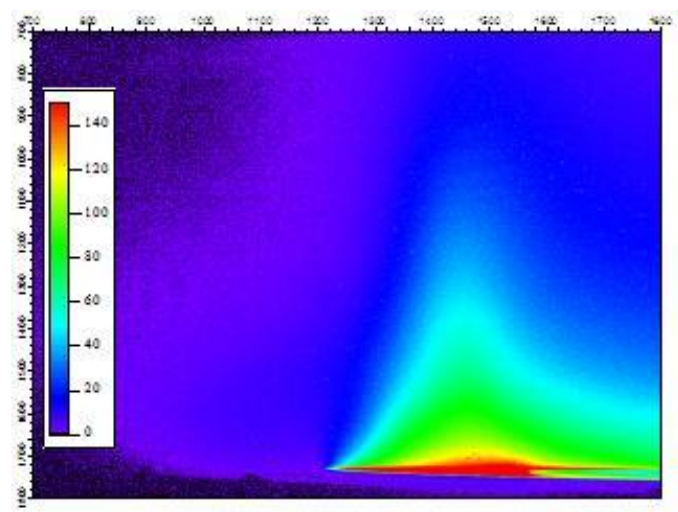

c. $\mathrm{J}_{\mathrm{D}}=75 \mathrm{~A}$

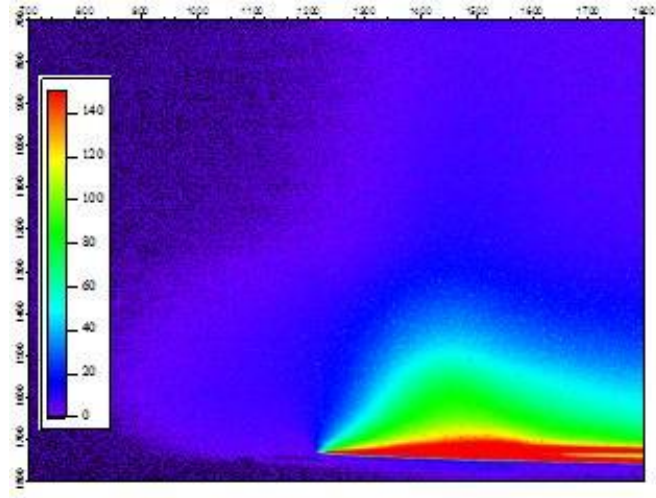

b. $\mathrm{J}_{\mathrm{D}}=50 \mathrm{~A}$

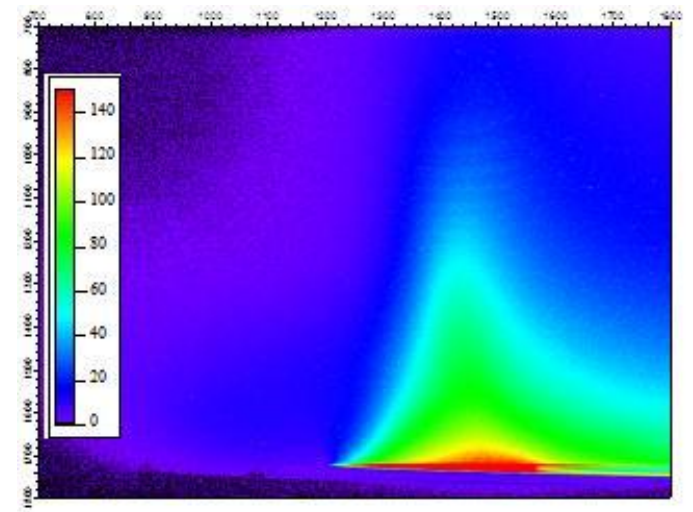

d. $\mathrm{J}_{\mathrm{D}}=100 \mathrm{~A}$

Figure 25 Contour plots of neutral images at the channel exit of the NASA $457 \mathrm{Mv} 2$ operating at $\mathrm{V}_{\mathrm{D}}=400 \mathrm{~V}$. 


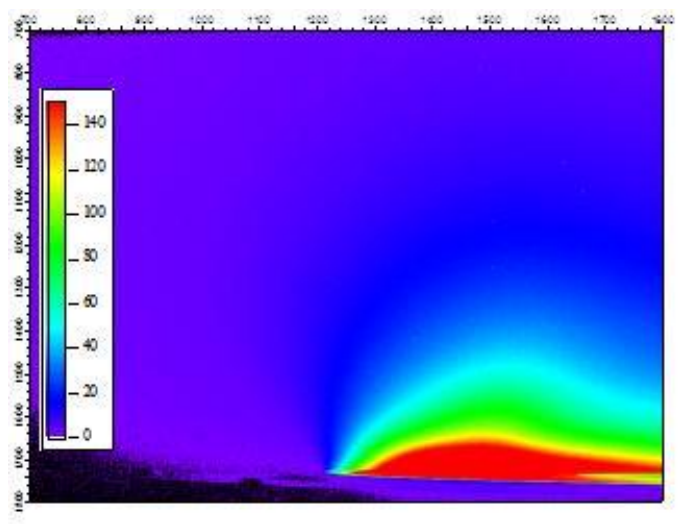

a $V_{D}=200 \mathrm{~V}$

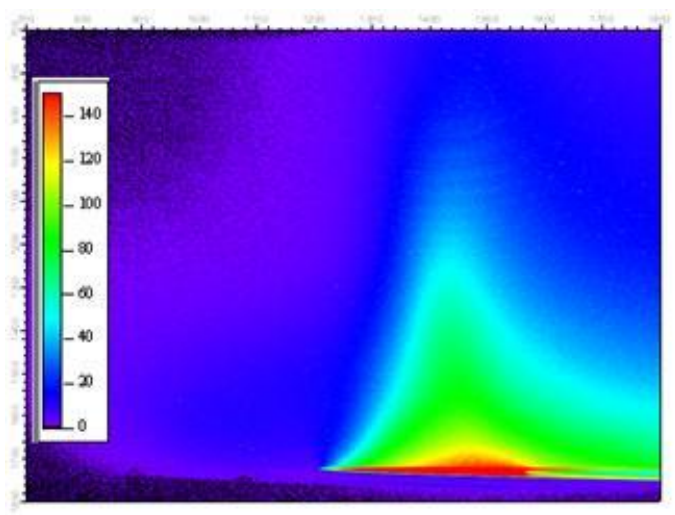

c. $\mathrm{V}_{\mathrm{D}}=400 \mathrm{~V}$

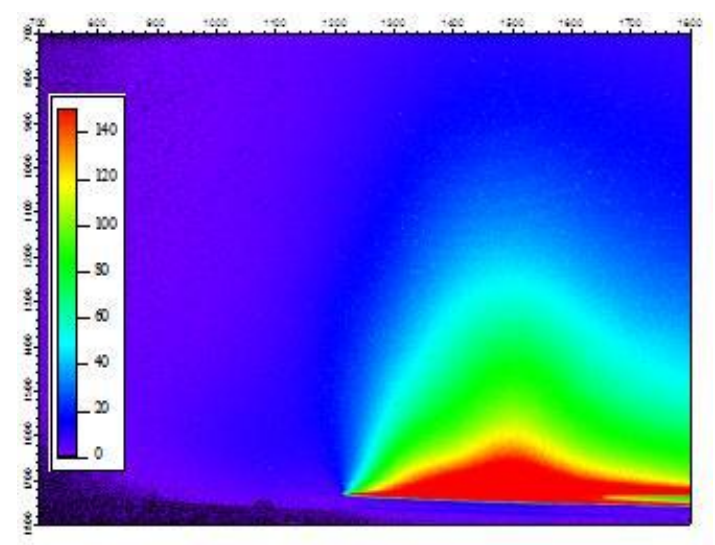

b. $\mathrm{V}_{\mathrm{D}}=300 \mathrm{~V}$

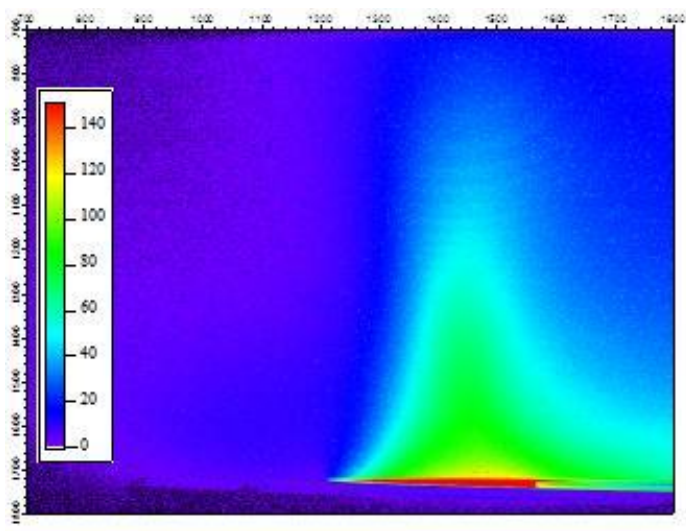

d. $V_{D}=500 \mathrm{~V}$

Figure 26 Contour plots of neutral images at the channel exit of the NASA 457Mv2 operating at $\mathrm{J}_{\mathrm{D}}=100 \mathrm{~A}$. 